\title{
Asymptotic behaviour of equicoercive diffusion energies in dimension two
}

\author{
Marc BRIANE \\ Centre de Mathématiques \\ I.N.S.A. de Rennes \& I.R.M.A.R. \\ mbriane@insa-rennes.fr
}

\author{
Juan CASADO-DÍAZ \\ Dpto. de Ecuaciones Diferenciales y Análisis Numérico \\ Universidad de Sevilla \\ jcasadod@us.es
}

November 3, 2006

\begin{abstract}
In this paper, we study the asymptotic behaviour of a given equicoercive sequence of diffusion energies $F_{n}, n \in \mathbb{N}$, defined in $L^{2}(\Omega)$, for a bounded open subset $\Omega$ of $\mathbb{R}^{2}$. We prove that, contrary to the three dimension (or greater), the $\Gamma$-limit of any convergent subsequence of $F_{n}$ is still a diffusion energy. We also provide an explicit representation formula of the $\Gamma$-limit when its domains contains the regular functions with compact support in $\Omega$. This compactness result is based on the uniform convergence satisfied by some minimizers of the equicoercive sequence $F_{n}$, which is specific to the dimension two. The compactness result is applied to the period framework, when the energy density is a highly oscillating sequence of equicoercive matrix-valued functions. So, we give a definitive answer to the question of the asymptotic behaviour of periodic conduction problems under the only assumption of equicoerciveness for the two-dimensional conductivity.
\end{abstract}

\section{Introduction}

This paper deals with the asymptotic behaviour of sequences of diffusion energies in a bounded open subset $\Omega$ of $\mathbb{R}^{2}$. The prototype of the diffusion energy is given by the following quadratic functional defined in $L^{2}(\Omega)$ :

$$
F_{n}(u):=\left\{\begin{array}{cl}
\int_{\Omega} A_{n} \nabla u \cdot \nabla u d x & \text { if } u \in H_{0}^{1}(\Omega) . \\
+\infty & \text { if } u \in L^{2}(\Omega) \backslash H_{0}^{1}(\Omega),
\end{array} \quad \text { for } n \in \mathbb{N},\right.
$$

where $A_{n}$ is a symmetric positive definite matrix-valued function in $L^{\infty}(\Omega)^{2 \times 2}$.

The knowledge of the limit behaviour of $F_{n}$ is crucial in the homogenization theory applied to conduction problems (see e.g. [1] for an introduction), since $A_{n}$ then represents the conductivity matrix of a given heterogeneous medium. In this context, Spagnolo [23], with the $G$-convergence theory, and Murat \& Tartar [25], [20], with the $H$-convergence theory, proved the compactness of the sequence $F_{n}$, when $A_{n}$ is assumed to be both equicoercive and equibounded. A few times later, Buttazzo \& Dal Maso [10] and Carbone \& Sbordone [12] extended the result of compactness by only assuming that the sequence $A_{n}$ is bounded and equiintegrable in $L^{1}(\Omega)^{2 \times 2}$. At the same period, Fenchenko \& Khruslov [15] showed that the equiintegrability condition cannot be relaxed since high conductivity regions in three dimension may induce nonlocal effects which correspond to a lack of compactness in the homogenization process (see also [2], [9], [4] for different approaches). 
Nonlocal effects naturally appear in the limit behaviour of the diffusion energy. Indeed, using the Beurling-Deny [3] theory Mosco [18] proved in particular that any sequence $F_{n}$ $\Gamma$-converges, up to a subsequence, for the strong topology of $L^{2}(\Omega)$ (see Definition 3.1) to a Dirichlet form (see Definition 3.3). According to the Beurling-Deny formula any Dirichlet form can always be split up into three terms: a strongly local form (the diffusion part), a local form and a nonlocal one. Inversely, Camar Eddine \& Seppecher [11] proved that any Dirichlet form in $L_{\text {loc }}^{2}\left(\mathbb{R}^{3}\right)$ can be obtained as the $\Gamma$-limit of a sequence of diffusion energies of type (1.1) with a suitable isotropic conductivity $A_{n}$.

The nonlocal effects obtained in the previous works are based on three-dimensional microstructures whose model example is a medium reinforced by a periodic lattice of high conductivity thin fibers. Then, it is natural to ask if the appearance of nonlocal effects is specific to the three dimension (or greater). Recently, in $\underline{[6]}$ for periodic microstructures and more generally in [7], we showed that the answer is positive. Assuming that the sequence $A_{n}$ is both equicoercive and bounded in $L^{1}(\Omega)^{2 \times 2}$, we proved that the $\Gamma$-limit of any sequence of type $F_{n}$ is a strongly local Dirichlet form. Therefore, the dimension two preserves the compactness in the homogenization process. The proof in $\underline{[6]},[7]$ is based on two-dimensional div-curl type lemmas which extend the one of Murat \& Tartar [26], [21]. Note that the equicoerciveness assumption is essential to obtain strongly convergent sequences in $L^{2}(\Omega)$. However, the use of div-curl lemmas is strictly limited to conductivity sequences which are bounded in $L^{1}(\Omega)^{2 \times 2}$.

In this paper, we study the asymptotic behaviour of the sequence of diffusion energies (1.1), without assuming any boundedness assumption on $A_{n}$. Our approach is completely different of the one used in $\underline{\underline{6]}}$ or [7] for $A_{n}$ bounded in $L^{1}(\Omega)^{2 \times 2}$. The keyingredient of the method is a uniform convergence result satisfied by some energy minimizers (see Section 2). More precisely, we prove (see Theorem 2.1) that for any bounded energy (with respect to $F_{n}$ ) sequence in $H^{1}(\Omega)$ which strongly converges in $L^{2}(\Omega)$ to a continuous function, there exists a smaller energy subsequence which strongly converges to the same limit in $L_{\mathrm{loc}}^{\infty}(\Omega)$. The proof of this result uses that the $p$-capicity, for $p \in(1,2)$, of a continuous curve is positive (see Lemma 2.8), which is specific to the dimension two. This combined with the continuity of the limit and the maximum principle allows us to construct a uniformly convergent sequence.

Up to our knowledge, the previous result provides new uniform estimates on solutions of uniformly elliptic partial differential equations without any control from above on the coefficients. Here, we give an example (see Corollary 2.5) of such an estimate for $A$-harmonic functions, where $A$ is any uniformly elliptic (but not necessarily uniformly bounded) matrix-valued in $L^{\infty}(O)$, for a bounded open subset $O$ of $\mathbb{R}^{2}$. This uniform estimate is used in the last section of the paper. More general cases are the subject of a work in progress [8].

On the other hand, thanks to the uniform convergence result of Section 2 and under the only assumption of equicoerciveness for $A_{n}$, we prove (see Section 3 and Theorem 3.6) that the diffusion energy $F_{n} \Gamma$-converges (up to a subsequence) for the strong topology of $L^{2}(\Omega)$ to a strongly local Dirichlet form $F$. Moreover, if the domain of the $\Gamma$-limit $F$ contains the space $C_{c}^{1}(\Omega)$ of the $C^{1}$-regular functions with compact support in $\Omega$, we obtain (see Theorem 3.4) the following representation formula

$$
F(u)=\int_{\Omega} A \nabla u \cdot \nabla u d \mu, \quad \forall u \in C_{c}^{1}(\Omega),
$$

where $\mu$ is a Radon measure on $\Omega$ and $A$ a matrix-valued function in $L_{\mu}^{\infty}(\Omega)^{2 \times 2}$. In other words, the sequence of the diffusion energies $F_{n}$ is relatively compact for the $L^{2}(\Omega)$ strong $\Gamma$-convergence topology in the set of the uniformly coercive diffusion energies. In particular, the compactness result implies that the limit of the energy density $A_{n} d x$ is still a density of type $A d \mu$. 
The compactness result of Section 3 has a remarkable application in the periodic homogenization framework. In this context, the conductivity $A_{n}$ is a highly oscillating sequence defined by $A_{n}(x):=B_{n}\left(\frac{x}{\varepsilon_{n}}\right)$, where $B_{n}$ is an equicoercive sequence of $(0,1)^{2}$ periodic matrix-valued functions in $L^{\infty}\left(\mathbb{R}^{2}\right)^{2 \times 2}$ and $\varepsilon_{n}$ is a positive sequence converging to zero. Associated with $B_{n}$ the constant matrix $A_{n}^{*}$ (see formula (4.4)) obtained, for a fixed $n$, from the periodic homogenization of $B_{n}\left(\frac{x}{\varepsilon}\right)$ as $\varepsilon \rightarrow 0$ (see e.g. [1]), plays a fundamental role in the homogenization process. Indeed, extending $[6]$ we prove (see Theorem 4.1) that the asymptotic behaviour of the diffusion energies (1.1) is completely determined by the limit behaviour of the matrix $A_{n}^{*}$, according to the following alternative:

- if the spectral radius $\rho\left(A_{n}^{*}\right)$ of $A_{n}^{*}$ is bounded, $A_{n}^{*}$ converges, up to a subsequence, to a matrix $A^{*}$ and the $\Gamma$-limit $F$ of $F_{n}$ satisfies (1.2) with the constant density $A^{*} d x$, in the whole space $H_{0}^{1}(\Omega)$;

- if $\rho\left(A_{n}^{*}\right)$ tends to $+\infty$, the domain of the $\Gamma$-limit reduces to $\{0\}$.

As an immediate consequence, the question on the asymptotic behaviour of the twodimensional periodic conduction problem

$$
\left\{\begin{array}{rlll}
-\operatorname{div}\left(B_{n}\left(\frac{x}{\varepsilon_{n}}\right) \nabla u_{n}\right) & = & f & \text { in } \Omega \\
u_{n} & = & 0 & \text { on } \partial \Omega,
\end{array} \quad \text { for } f \in H^{-1}(\Omega),\right.
$$

is now definitively solved under the only assumption of equicoerciveness for $B_{n}$ :

- if $\rho\left(A_{n}^{*}\right)$ is bounded, (1.3) converges, up to a subsequence, to the conduction problem with the constant conductivity $\lim _{n \rightarrow+\infty} A_{n}^{*}$;

- if $\rho\left(A_{n}^{*}\right)$ tends to $+\infty$, the potential $u_{n}$ of (1.3) strongly converges to zero in $H_{0}^{1}(\Omega)$.

The paper is organized as follows. Section 2 is devoted to the uniform convergence results and Section 3 to the $\Gamma$-convergence of sequences of diffusion energies of type (1.1). In Section 4 we apply the results of Section 3 to the periodic framework.

\section{Notations}

- $\mathbb{N}^{*}:=\mathbb{N} \backslash\{0\}$ denotes the set of the positive integers;

- $a \vee b$, resp. $a \wedge b$, denotes the maximum, resp. the minimum, of $a, b \in \mathbb{R}$;

- $B\left(x_{0}, \delta\right)$ denotes the disk of center $x_{0} \in \mathbb{R}^{2}$ and of radius $\delta>0$;

- $\chi_{E}$ denotes the characteristic of the set $E$;

- $\exists \lim$ means that the limit does exist;

- $\Omega$ denotes an open subset of $\mathbb{R}^{2}$ and $\bar{\Omega}$ the closure of $\Omega$ in $\mathbb{R}^{2}$;

- $H_{\text {loc }}(\Omega)$ means locally in the space $H(\Omega)$;

- $C(\Omega)$ denotes the space of the continuous functions in $\Omega, C_{0}(\Omega)$ the subspace of $C(\Omega)$ composed of the functions which are zero on the boundary of $\Omega, C_{c}(\Omega)$ the subspace of $C_{0}(\Omega)$ composed of the functions with compact support in $\Omega$, and $C_{c}^{k}(\Omega)$, for $k \in$ $\mathbb{N} \cap\{+\infty\}$, the subspace of $C_{c}(\Omega)$ composed of the $k$-th continuously differentiable functions in $\Omega$;

- $\mathcal{D}^{\prime}(\Omega)$ denotes the set of the distributions on $\Omega$;

- $\mathcal{M}(\Omega)$ denotes the set of the Radon measures on $\Omega$; 
- a sequence $\mu_{n}$ in $\mathcal{M}(\Omega)$ converges to $\mu \in \mathcal{M}(\Omega)$ in the weak $*$ sense of the measures in $\Omega$ if

$$
\lim _{n \rightarrow+\infty} \int_{\Omega} \varphi d \mu_{n}=\int_{\Omega} \varphi d \mu, \quad \forall \varphi \in C_{0}(\Omega)
$$

and the convergence is denoted by $\mu_{n} \rightarrow \mu$ in $\mathcal{M}(\Omega) *$;

- q.e. means quasi-everywhere in the sense of the 2-capacity in $\mathbb{R}^{2}$, and a.e. means everywhere in the sense of the Lebesgue measure in $\mathbb{R}^{2}$;

- for any $p \in(1,2)$ and for any subset $E$ of $\mathbb{R}^{2}, C_{p}(E)$ denotes the $p$-capacity of $E$ with respect to $\mathbb{R}^{2}$, which is defined by

$$
C_{p}(E):=\inf \left\{\int_{\mathbb{R}^{2}}|\nabla u|^{p} d x: u \in D^{1, p}\left(\mathbb{R}^{2}\right), u \geq 1 \text { a.e. in a neighbourhood of } E\right\},
$$

where $D^{1, p}\left(\mathbb{R}^{2}\right)$ is the space of the functions $u$ in $L^{\frac{2 p}{2-p}}\left(\mathbb{R}^{2}\right)$ such that $\nabla u \in L^{2}\left(\mathbb{R}^{2}\right)^{2}$.

\section{Uniform convergence results}

\subsection{Statement of the results}

Let $\Omega$ be an open subset of $\mathbb{R}^{2}$. In this section, we consider a given sequence of symmetric matrix-valued functions $A_{n} \in L^{\infty}(\Omega)^{2 \times 2}, n \in \mathbb{N}$, which satisfies the following equicoerciveness property in $\Omega$

$$
\exists \alpha>0 \quad \text { such that } \quad \forall n \in \mathbb{N}, \forall \xi \in \mathbb{R}^{2}, \quad A_{n} \xi \cdot \xi \geq \alpha|\xi|^{2} \quad \text { a.e. in } \Omega .
$$

For any function $u \in H^{1}(\Omega) \cap C(\Omega)$, we will study some questions related to the existence of sequences $u_{n}$ in $H^{1}(\Omega)$ which both converge uniformly to $u$ in $\Omega$ and satisfy the following minimization property

$$
\exists \lim _{n \rightarrow+\infty} \int_{\Omega} A_{n} \nabla u_{n} \cdot \nabla u_{n} d x \leq \liminf _{n \rightarrow+\infty} \int_{\Omega} A_{n} \nabla v_{n} \cdot \nabla v_{n} d x
$$

for any sequence $v_{n}$ in $H^{1}(\Omega)$ (some boundary conditions can be added), which strongly converges to $u$ in $L^{2}(\Omega)$. Our main result in this way is the following theorem:

Theorem 2.1. Let $u$ be a function in $H^{1}(\Omega) \cap C(\Omega)$ and let $\hat{u}_{n}$ be a sequence $H^{1}(\Omega)$ which strongly converges to $u$ in $L^{2}(\Omega)$ and satisfies

$$
\exists \lim _{n \rightarrow+\infty} \int_{\Omega} A_{n} \nabla \hat{u}_{n} \cdot \nabla \hat{u}_{n} d x<+\infty .
$$

Then, up to a subsequence of $n$, still denoted by $n$, there exists $u_{n} \in H^{1}(\Omega)$ which satisfies

$$
\begin{gathered}
\limsup _{n \rightarrow+\infty} \int_{\Omega} A_{n} \nabla u_{n} \cdot \nabla u_{n} d x \leq \lim _{n \rightarrow+\infty} \int_{\Omega} A_{n} \nabla \hat{u}_{n} \cdot \nabla \hat{u}_{n} d x, \\
\text { and } \quad u_{n} \longrightarrow u \text { strongly in } L_{\mathrm{loc}}^{\infty}(\Omega) .
\end{gathered}
$$

Moreover, if the support of $u$ is contained in a compact set $K$ of $\Omega$, then we can take $u_{n}$ such that $u_{n}=0$ q.e. in $\Omega \backslash K$, for any $n \in \mathbb{N}$.

Remark 2.2. If in Theorem 2.1 the sequence $\hat{u}_{n}$ is in $H_{0}^{1}(\Omega)$ and $u$ in $H_{0}^{1}(\Omega) \cap C_{0}(\Omega)$, then we can choose $u_{n}$ in $H_{0}^{1}(\Omega)$, which strongly converges to $u$ in $L^{\infty}(\Omega)$. To this end, it is enough to consider a bounded open set $\tilde{\Omega}$ such that $\bar{\Omega} \subset \tilde{\Omega}$ and to apply the second part of Theorem 2.1 to the sequences $\tilde{u}_{n}$ and $\tilde{A}_{n}$ defined by

$$
\tilde{u}_{n}(x):=\left\{\begin{array}{ll}
u_{n}(x) & \text { if } x \in \Omega \\
0 & \text { if } x \in \tilde{\Omega} \backslash \Omega,
\end{array} \quad \text { and } \quad \tilde{A}_{n}(x):= \begin{cases}A_{n}(x) & \text { if } x \in \Omega \\
I_{2} & \text { if } x \in \tilde{\Omega} \backslash \Omega .\end{cases}\right.
$$


Corollary 2.3. Consider $\hat{u}_{n} \in H^{1}(\Omega)$ and $u \in H^{1}(\Omega) \cap C(\Omega)$ such that

$$
\hat{u}_{n} \rightarrow u \quad \text { weakly in } H_{\mathrm{loc}}^{1}(\Omega) \quad \text { and } \quad \operatorname{div}\left(A_{n} \nabla \hat{u}_{n}\right)=0 \quad \text { in } \mathcal{D}^{\prime}(\Omega) \text {. }
$$

Then, we have the following uniform convergence

$$
\hat{u}_{n} \longrightarrow u \quad \text { strongly in } C(\Omega) \text {. }
$$

Remark 2.4. Note that in Corollary 2.3 each function $u_{n}$ is continuous in $\Omega$ by the De Giorgi-Stampacchia theorem (see e.g. [16] Chapter 8).

Corollary 2.5. For any open subset $\Omega$ of $\mathbb{R}^{2}$ and any compact subset $K$ of $\Omega$, there exists a constant $C>0$ which only depends on $\Omega$ and $K$ such that, for any matrix-valued function $A \in L^{\infty}(\Omega)^{2 \times 2}$ satisfying the uniform coerciveness (2.1) and any function $u \in H^{1}(\Omega)$ solution of

$$
\operatorname{div}(A \nabla u)=0 \quad \text { in } \mathcal{D}^{\prime}(\Omega),
$$

the following estimate holds true

$$
\|u\|_{C(K)} \leq C\|u\|_{H^{1}(\Omega)}
$$

Remark 2.6. Corollaries 2.3 and 2.5 can be deduced from [8] where more general results, for non-necessarily homogeneous equations, are proved.

\subsection{Proof of the results}

Let us now give the proof of the uniform convergence results stated in the previous section. We will need the two following lemmas:

Lemma 2.7. Let $O$ be a bounded open subset of $\mathbb{R}^{2}$ and let $u \in H^{1}(O) \cap C(\bar{O})$. Denote $M:=\max _{\partial O} u$ and $m:=\min _{\partial O} u$.

Then, for any function $v$ such that $v-u$ belongs to $H_{0}^{1}(O)$, the functions $(v-M)^{+}$and $(m-v)^{+}$belong to $H_{0}^{1}(O)$.

Proof. Consider $\varphi_{\varepsilon} \in C_{c}^{\infty}(O)$, for $\varepsilon>0$, which strongly converges to $v-u$ in $H_{0}^{1}(\Omega)$ as $\varepsilon \rightarrow 0$. Since $u$ is continuous in $\bar{O}$, the functions $U_{\varepsilon}:=\left(u+\varphi_{\varepsilon}-M-\varepsilon\right)^{+}$and $u_{\varepsilon}:=\left(m-\varepsilon-u-\varphi_{\varepsilon}\right)^{+}$have compact support in $O$. The functions $U_{\varepsilon}$ and $u_{\varepsilon}$ belong to $H^{1}(O)$, hence they also belong to $H_{0}^{1}(O)$. Therefore, using that $U_{\varepsilon}$ and $u_{\varepsilon}$ strongly converge respectively to $(v-M)^{+}$and $(m-v)^{+}$in $H^{1}(O)$, yields the result.

Lemma 2.8. For any $p \in(1,2)$ and any continuous curve $L$ of extremities $a, b$, we have

$$
C_{p}(L) \geq R_{p}|a-b|^{2-p},
$$

where $R_{p}>0$ is the $p$-capacity of a unit segment in $\mathbb{R}^{2}$.

Proof. Using a translation, a rotation and a homothety, we can reduce the proof to the case where $a=(0,0), b=(1,0)$. Then, consider a curve $L$ of extremities $(0,0),(1,0)$ and take a function $\varphi \in C_{c}^{\infty}\left(\mathbb{R}^{2}\right)$ such that $\varphi>\chi_{L}$. By the Pólya-Szegö inequality [22] extended to any power $p \geq 1$ (see e.g. [24] and Chapter I.4 of [19]), it is known that the Steiner symmetrization $\varphi^{*}$ of $\varphi$ with respect to $\left\{x_{2}=0\right\}$, defined by its level sets

$$
\left\{\left(x_{1}, x_{2}\right) \in \mathbb{R}^{2}: \varphi^{*}\left(x_{1}, x_{2}\right)>c\right\}=\left\{\left(x_{1}, x_{2}\right) \in \mathbb{R}^{2}:\left|x_{2}\right|<\frac{1}{2}\left|\left\{y \in \mathbb{R}: \varphi\left(x_{1}, y\right)>c\right\}\right|\right\},
$$


belongs to $W_{c}^{1, p}\left(\mathbb{R}^{2}\right)$ and satisfies

$$
\int_{\mathbb{R}^{2}}\left|\nabla \varphi^{*}\right|^{p} d x \leq \int_{\mathbb{R}^{2}}|\nabla \varphi|^{p} d x
$$

Moreover, since $L$ and $\varphi$ are continuous, it is clear that $\varphi^{*}>1$ in $[0, \underline{1}] \times\{0\}$, hence

$$
C_{p}\left([0, \underline{1]} \times\{0\}) \leq \int_{\mathbb{R}^{2}}\left|\nabla \varphi^{*}\right|^{p} d x \leq \int_{\mathbb{R}^{2}}|\nabla \varphi|^{p} d x .\right.
$$

Taking the infimum in $\varphi$, we get the desired estimate (2.6).

Proof of Theorem 2.1. Using the density of $H^{1}(\Omega) \cap C^{\infty}(\Omega)$ in $H^{1}(\Omega)$ (see e.g. [16]), we can also assume that $\hat{u}_{n}$ is continuous in $\Omega$. For any $\delta>0$, we define $\Omega_{\delta}$ by

$$
\Omega_{\delta}:=\{x \in \Omega: d(x, \partial \Omega)>\delta\} .
$$

Since $u$ is continuous in $\bar{\Omega}_{\delta}$, for any $l \in \mathbb{N}^{*}$, there exists $\delta_{l}>0$, with $\lim _{l \rightarrow+\infty} \delta_{l}=0$, such that

$$
|u(x)-u(y)|<\frac{1}{2 l}, \quad \forall x, y \in \bar{\Omega}_{\delta_{l}}, \text { with }|x-y| \leq \delta_{l} .
$$

Let $p \in(1,2)$. Since $\hat{u}_{n}$ weakly converges in $H^{1}(\Omega)$, there exists (see e.g. [14]) a subsequence of $\hat{u}_{n}$, still denoted by $\hat{u}_{n}$, which converges to $u C_{p}$-quasi uniformly in every open set $\omega \subset \Omega$, with $\bar{\omega} \subset \Omega$ ( $\omega$ can be chosen as $\Omega$ if $\Omega$ is smooth). Thus, we can choose this sequence in such a way that for any $l \in \mathbb{N}^{*}$, there exists a relatively closed subset $K_{l}$ of $\Omega$ satisfying

$$
\begin{gathered}
C_{p}\left(\Omega \backslash K_{l}\right)<R_{p} \delta_{l}^{2-p}, \\
\left|\hat{u}_{n}(x)-u(x)\right|<\frac{1}{2 l}, \quad \forall x \in \Omega_{\delta_{l}} \cap K_{l}, \forall n \geq l .
\end{gathered}
$$

Then, we define $u_{n}$ by

$$
\left\{\begin{array}{l}
u_{n}:=\hat{u}_{n} \text { in } K_{l}, \quad u_{n}-\hat{u}_{n} \in H_{0}^{1}\left(\Omega \backslash K_{l}\right), \\
\int_{\Omega \backslash K_{l}} A_{n} \nabla u_{n} \cdot \nabla u_{n} d x \leq \int_{\Omega \backslash K_{l}} A_{n} \nabla v \cdot \nabla v d x, \quad \forall v, v-\hat{u}_{n} \in H_{0}^{1}\left(\Omega \backslash K_{l}\right) .
\end{array}\right.
$$

Clearly, $u_{n}$ satisfies $(2.2)$. Let us prove that $u_{n}$ strongly converges to $u$ in $L_{\text {loc }}^{\infty}(\Omega)$. To this end, we fix $\delta>0$. We have

$$
\left|u_{n}(x)-u(x)\right|=\left|\hat{u}_{n}(x)-u(x)\right|<\frac{1}{2 l}, \quad \forall x \in \Omega_{\delta} \cap K_{l}, \forall n \geq l, \text { with } \delta_{l}<\delta .
$$

Consider a connected component $O$ of $\Omega \backslash K_{l}$ such that $O \cap \Omega_{\delta} \neq \varnothing$. Since $O$ is opened and connected, it is connected by curves. Thus, for any $y_{1}, y_{2} \in O$, there exists a curve $L \subset O$ which connects $y_{1}, y_{2}$. By Lemma 2.8 and (2.8), we have

$$
R_{p}\left|y_{1}-y_{2}\right|^{2-p} \leq C_{p}(L) \leq C_{p}(O) \leq C_{p}\left(\Omega \backslash K_{l}\right) \leq R_{p} \delta_{l}^{2-p},
$$

hence diam $(O) \leq \delta_{l}$. Then, taking $l$ large enough such that $2 \delta_{l}<\delta$, we get that $\bar{O} \subset \Omega_{\delta_{l}}$, and in particular, $\partial O \subset \Omega_{\delta_{l}} \cap K_{l}$. Denote

$$
m_{n}:=\min _{\partial O} \hat{u}_{n} \quad \text { and } \quad M_{n}:=\max _{\partial O} \hat{u}_{n}
$$

By Lemma $2.7\left(u_{n}-M_{n}\right)^{+}$and $\left(m_{n}-u_{n}\right)^{-}$belong to $H_{0}^{1}(O)$, and by definition $(2.10) u_{n}$ is $A_{n}$-harmonic in $O$. Then, the maximum principle yields

$$
m_{n} \leq u_{n} \leq M_{n}, \text { q.e. in } O, \forall n \in \mathbb{N} .
$$


On the other hand, since $\partial O \subset \Omega_{\delta_{l}} \cap K_{l}$, we have by (2.9).

$$
m_{n} \geq \min _{\partial O} u-\frac{1}{2 l} \quad \text { and } \quad M_{n} \leq \max _{\partial O} u+\frac{1}{2 l}, \quad \forall n \geq l .
$$

Moreover, $\bar{O} \subset \Omega_{\delta_{l}}$, $\operatorname{diam}(O) \leq \delta_{l}$ and (2.7) imply that

$$
\min _{\partial O} u \geq u(x)-\frac{1}{2 l} \quad \text { and } \quad \max _{\partial O} u \leq u(x)+\frac{1}{2 l}, \quad \forall x \in O, \forall n \geq l .
$$

Therefore, (2.12) combined with the two previous estimates yields

$$
\left|u_{n}-u\right| \leq \frac{1}{2 l}, \quad \text { q.e. in } O,
$$

hence the sequence $u_{n}$ satisfies the uniform convergence (2.3).

Now, assume that the support of $u$ is contained in a compact subset $K$ of $\Omega$, and consider an open set $\tilde{\Omega}$ which contains $K$ and is strictly contained in $\Omega$. For any $\varepsilon>0$, let $S_{\varepsilon}$ be the function defined by $\left.S_{\varepsilon}(s):=(s-\varepsilon \operatorname{sgn}(s))\right) \chi_{\{|s|>\varepsilon\}}$, for $s \in \mathbb{R}$. Since $u_{n}$ strongly converges to $u$ in $L^{\infty}(\tilde{\Omega})$, the sequence $\varepsilon_{n}:=\left\|u_{n}-u\right\|_{L^{\infty}(\tilde{\Omega})}$ tends to zero. Therefore, the sequence $\tilde{u}_{n}:=\chi_{\tilde{\Omega}} S_{\varepsilon_{n}}\left(u_{n}\right)$ satisfies conditions (2.2), (2.3) and vanish q.e. in $\Omega \backslash K$.

Proof of Corollary 2.3. Since $\hat{u}_{n}$ satisfies $\operatorname{div}\left(A_{n} \nabla \hat{u}_{n}\right)=0$ in $\mathcal{D}^{\prime}(\Omega)$, it is Hölder continuous in $\Omega$ by the De Giorgi-Stampacchia theorem. Then, the argument used in the proof of Theorem 2.1 proves that the sequence $u_{n}$ defined by (2.10) strongly converges to $u$ in $L_{\text {loc }}^{\infty}(\Omega)$. However, we have $u_{n}=\hat{u}_{n}$ by construction, which yields the desired result.

Proof of Corollary 2.5. We reason by contradiction. If the result does not hold true, then, for any $n \in \mathbb{N}$, there exist $u_{n} \in H^{1}(\Omega), A_{n} \in L^{\infty}(\Omega)^{2 \times 2}$ and $\gamma_{n}>0$ such that

$$
\begin{gathered}
A_{n} \xi \cdot \xi \geq \gamma_{n}|\xi|^{2}, \quad \forall \xi \in \mathbb{R}^{2}, \quad \text { a.e. } x \in \Omega, \\
\text { and } \quad\left\|u_{n}\right\|_{C(K)}>n\left\|u_{n}\right\|_{H^{1}(\Omega)} .
\end{gathered}
$$

Up to replace $A_{n}$ by $A_{n} / \gamma_{n}$ and $u_{n}$ by $u_{n} /\left\|u_{n}\right\|_{C(K)}$, we can assume that $\gamma_{n}=1$ and $\left\|u_{n}\right\|_{C(K)}=1$. Then, $A_{n}$ is equicoercive and by (2.13) $u_{n}$ strongly converges to zero in $H^{1}(\Omega)$. Therefore, by Corollary $2.3 u_{n}$ converges uniformly to zero in $K$, in contradiction with $\left\|u_{n}\right\|_{C(K)}=1$.

\section{$3 \quad \Gamma$-limit of equicoercive diffusion energies}

\section{1 $\Gamma$-convergence and Dirichlet forms}

In this section we first recall the definition of the De Giorgi $\Gamma$-convergence and some of its properties which will be used in the sequel. We refer to [13] for an exhaustive presentation of the $\Gamma$-convergence.

Definition 3.1. A sequence of functionals $F_{n}: L^{2}(\Omega) \longrightarrow[0,+\infty]$ is said to $\Gamma$-converge to $F: L^{2}(\Omega) \longrightarrow[0,+\infty]$ for the strong topology of $L^{2}(\Omega)$ if, for any $u$ in $L^{2}(\Omega)$,

(i) the $\Gamma$-liminf inequality holds

$$
\forall u_{n} \longrightarrow u \text { strongly in } L^{2}(\Omega), \quad F(u) \leq \liminf _{n \rightarrow+\infty} F_{n}\left(u_{n}\right),
$$


(ii) the $\Gamma$-limsup inequality holds

$$
\exists \bar{u}_{n} \longrightarrow u \text { strongly in } L^{2}(\Omega), \quad F(u)=\lim _{n \rightarrow+\infty} F_{n}\left(\bar{u}_{n}\right) .
$$

Any sequence satisfing (3.2) will be called a recovery sequence for $F_{n}$, of limit $u$.

In the sequel, we will always consider the $\Gamma$-convergence with respect to the strong topology of $L^{2}$. Consequently, this topology will be not necessarily mentionned.

\section{Properties 3.2.}

a) Since $L^{2}(\Omega)$ is separable, any sequence of functionals $F_{n}: L^{2}(\Omega) \longrightarrow[0,+\infty]$ has a subsequence which $\Gamma$-converges with respect to the strong topology of $L^{2}(\Omega)$.

b) Let $F_{n}: L^{2}(\Omega) \longrightarrow[0,+\infty]$ be a sequence of quadratic forms which $\Gamma$-converges to $F$. Then, $F$ is a quadratic form on $L^{2}(\Omega)$ which is semi-lower continuous with respect to the strong topology of $L^{2}(\Omega)$.

c) Let $F_{n}: L^{2}(\Omega) \longrightarrow[0,+\infty]$ be a sequence of quadratic forms which $\Gamma$-converges to $F$. Let $\Phi_{n}, \Phi$ be the polar forms respectively associated with $F_{n}, F$ on their domains. Then, for any $u \in L^{2}(\Omega)$, with $F(u)<+\infty$, a sequence $u_{n}$ in $L^{2}(\Omega)$ is a recovery sequence $(3.2)$ for $F_{n}$, of limit $u$, if and only if

$$
\forall v_{n} \longrightarrow v \text { strongly in } L^{2}(\Omega) \text {, with } F_{n}\left(v_{n}\right) \leq c, \quad \lim _{n \rightarrow+\infty} \Phi_{n}\left(u_{n}, v_{n}\right)=\Phi(u, v),
$$

or equivalently, (3.3) with $v=0$.

Now, we recall some notions about Dirichlet forms, which will be used in the statement of Theorem 3.8. We refer to $\underline{[18]}$ for more details in connection with the $\Gamma$-convergence.

Definition 3.3. Let $X$ be a Hausdorff, separable, locally compact space, and let $m$ be a $\sigma$-finite nonnegative Radon measure on $X$. Let $H$ be the space $L_{m}^{2}(X)$ endowed with its Hilbert norm $\|\cdot\|_{H}$. Let $F: H \longrightarrow[0,+\infty]$ be a quadratic form of domain $D(F):=$ $\{u \in H: F(u)<+\infty\}$, whose polar form $\Phi$ is a bilinear form defined in $D(F) \times D(F)$.

(i) The form $F$ is said to be closed if it is semi-lower continuous with respect to the norm $\|\cdot\|_{H}$. The form $F$ is said to be closable if there exists an extension $\tilde{F}$ of $F$ in $H$ such that $D(F) \subset D(\tilde{F})$. The closure of a closable form is its smallest closed extension in $H$.

(ii) The form $F$ is said to be Markovian if

$$
\forall u \in D(F), \quad v:=(u \vee 0) \wedge 1 \in D(F) \text { and } \quad F(v) \leq F(u) .
$$

(iii) A Dirichlet form on $H$ is a closed Markovian quadratic form defined in $H$.

(iv) The form $F$ is said to be regular if there exists a subset of $D(F) \cap C_{0}(X)$, which is dense both in $C_{0}(X)$ with the uniform norm and in $D(F)$ with the norm $\left(F+\|\cdot\|_{H}\right)^{1 / 2}$.

(v) The form $F$ is said to be local if

$$
\Phi(u, v)=0, \quad \forall u, v \in D(F), \text { with } \operatorname{supp}(u) \cap \operatorname{supp}(v)=\varnothing .
$$

The form $F$ is said to be strongly local if

$$
\Phi(u, v)=0, \quad \forall u, v \in D(F), \text { with } u=\operatorname{cst} \text { in } \operatorname{supp}(v) .
$$

Thanks to the Beurling-Deny theory [3] any regular Dirichlet form $F$ on $L_{m}^{2}(X)$ can be split up on its domain into three specific forms: a strongly local form $F_{d}$, a local form and a nonlocal one. More precisely, the following representation formula holds for any $u \in D(F)$,

$$
F(u)=F_{d}(u)+\int_{X} u^{2}(x) k(d x)+\iint_{X \times X \backslash \operatorname{diag}}(u(x)-u(y))^{2} j(d x, d y),
$$

where $F_{d}$ is called the diffusion part of $F, k$ the killing measure and $j$ the jumping measure. 


\subsection{Statement of the results}

As in Section 2, let us consider a bounded open subset $\Omega$ of $\mathbb{R}^{2}$, and a sequence of symmetric matrix-valued functions $A_{n} \in L^{\infty}(\Omega)^{2 \times 2}$ which satisfy $(2.1)$. For any $n \in \mathbb{N}$ and any open subset $\omega$ of $\Omega$, we define the quadratic form $F_{n}(\cdot, \omega)$ in $L^{2}(\omega)$ by

$$
F_{n}(u, \omega):=\left\{\begin{array}{cl}
\int_{\omega} A_{n} \nabla u \cdot \nabla u d x & \text { if } u \in H_{0}^{1}(\omega) . \\
+\infty & \text { if } u \in L^{2}(\omega) \backslash H_{0}^{1}(\omega) .
\end{array}\right.
$$

The form $F_{n}(\cdot, \Omega)$ is simply denoted by $F_{n}$.

Assume that $F_{n} \Gamma$-converges to some quadratic form $F$ for the topology of $L^{2}(\Omega)$, which holds true for a subsequence in virtue of Properties $3.2 a$ ). Since $F_{n}$ is clearly Markovian, the properties (3.1), (3.2) of the $\Gamma$-convergence imply that $F$ is also Markovian. Moreover, thanks to Properties $3.2 b) F$ is closed. Therefore, $F$ is a Dirichlet form in the sense of Definition $3.3(\mathrm{iii})$. The following result gives a necessary and sufficient condition to have $F$ regular with $C_{c}^{1}(\Omega) \subset D(F)$. When this condition is satisfied, $F$ is a strongly local (3.4) Dirichlet form whose integral representation for regular functions is independent of the domain.

Theorem 3.4. The domain $D(F)$ of $F$ contains $C_{c}^{1}(\Omega)$ if and only if, for any $x_{0} \in$ $\Omega$, there exists $\delta>0$, two functions $w^{1}, w^{2}$ in $C^{1}\left(B\left(x_{0}, \delta\right)\right)$ and two sequences $w_{n}^{1}, w_{n}^{2}$ in $H^{1}\left(B\left(x_{0}, \delta\right)\right), n \in \mathbb{N}$, such that

$$
\begin{cases}B\left(x_{0}, \delta\right) \subset \Omega, & \\ \nabla w^{1}\left(x_{0}\right), \nabla w^{2}\left(x_{0}\right) \text { are linearly independent, } & \\ w_{n}^{i} \longrightarrow w^{i} \text { strongly in } L^{2}\left(B\left(x_{0}, \delta\right)\right), & \text { for } i=1,2, \\ A_{n} \nabla w_{n}^{i} \cdot \nabla w_{n}^{i} \text { is bounded in } L^{1}\left(B\left(x_{0}, \delta\right)\right), & \text { for } i=1,2 .\end{cases}
$$

Assume that $C_{c}^{1}(\Omega)$ is contained in $D(F)$. Then, there exist a nonnegative Radon measure $\mu$ on $\Omega$ and a nonnegative matrix-valued function $A$ in $L_{\mu}^{\infty}(\Omega)^{2 \times 2}$ such that the regular part $A^{r}$ of Ad $\mu$ with respect to the Lebesgue measure satisfies

$$
A^{r} \xi \cdot \xi \geq \alpha|\xi|^{2}, \quad \forall \xi \in \mathbb{R}^{2} \text {, a.e. in } \Omega,
$$

and such that, for any open set $\omega$ of $\Omega$, the $\Gamma$-limit $F(\cdot, \omega)$ of $F_{n}(., \omega)$ with respect to the strong topology of $L^{2}(\omega)$ does exist on $C_{c}^{1}(\omega)$ and reads as

$$
F(u, \omega)=\int_{\omega} A \nabla u \cdot \nabla u d \mu, \quad \forall u \in C_{c}^{1}(\omega) .
$$

Moreover, for any $u \in C_{c}^{1}(\omega)$ and any $u_{n} \in H_{0}^{1}(\omega)$ which strongly converges to $u$ in $L^{2}(\omega)$ and such that $F_{n}\left(u_{n}, \omega\right)$ tends to $F(u, \omega)$, the sequence $A_{n} \nabla u_{n} \cdot \nabla u_{n}$ converges to $A \nabla u$. $\nabla u d \mu$ in the weak $*$ sense of the measures in $\omega$.

Remark 3.5. In the second part of Theorem 3.4 the $\Gamma$-convergence of $F_{n}(\cdot, \omega)$ to $F(\cdot, \omega)$ holds true up to a subsequence which does depend on the open set $\omega$. However, the integral representation (3.9) of $F(u, \omega)$, which is valid on $C_{c}^{1}(\omega)$, is independent of $\omega$.

In fact, the integral expression (3.9) holds true for any $u \in C_{0}^{1}(\omega)$, with $A \nabla u \cdot \nabla u \in$ $L_{\mu}^{1}(\omega)$. Indeed, it is easy to check that these functions can be approximated by functions in $C_{c}^{1}(\omega)$ in the strong topology of $D(F(., \omega))$.

Theorem 3.4 provides an integral representation of $F$, assuming that $D(F)$ contains $C_{c}^{1}(\Omega)$. The following result gives a corrector result: 
Theorem 3.6. Assume that $D(F)$ contains $C_{c}^{1}(\Omega)$. Then, there exists a sequence $\left(w_{n}^{1}, w_{n}^{2}\right)$ in $H_{0}^{1}\left(\Omega ; \mathbb{R}^{2}\right) \cap L^{\infty}\left(\Omega ; \mathbb{R}^{2}\right)$ which strongly converges to the identity in $L_{\mathrm{loc}}^{\infty}\left(\Omega ; \mathbb{R}^{2}\right)$, and which satisfies, for any $i \in\{1,2\}$ and any compact subset $K$ of $\Omega$,

$$
\begin{gathered}
\limsup _{n \rightarrow+\infty} \int_{K} A_{n} \nabla w_{n}^{i} \cdot \nabla w_{n}^{i} d x<+\infty, \\
\lim _{n \rightarrow+\infty} \int_{K} A_{n} \nabla w_{n}^{i} \cdot \nabla v_{n} d x=0, \quad\left\{\begin{array}{l}
\forall v_{n} \in H_{0}^{1}(\Omega), v_{n}=0 \text { q.e. in } \Omega \backslash K, \\
v_{n} \rightarrow 0 \text { in } L^{2}(\Omega), A_{n} \nabla v_{n} \cdot \nabla v_{n} \text { bounded in } L^{1}(\Omega) .
\end{array}\right.
\end{gathered}
$$

Let $\omega$ be open subset of $\Omega$ and let $u \in C_{c}^{1}(\omega)$. Assume that the sequence $F_{n}(\cdot, \omega) \Gamma$ converges to $F(\cdot, \omega)$ for the strong topology of $L^{2}(\omega)$. Then, for any recovery sequence $u_{n} \in H_{0}^{1}(\omega)$ which is zero q.e. outside a compact subset of $\omega$, which strongly converges to $u$ in $L^{2}(\omega)$ and such that $F_{n}\left(u_{n}, \omega\right)$ tends to $F(u, \omega)$, we have

$$
\lim _{n \rightarrow+\infty} \int_{\omega} A_{n}\left(\nabla u_{n}-\sum_{i=1}^{2} \partial_{i} u \nabla w_{n}^{i}\right) \cdot\left(\nabla u_{n}-\sum_{i=1}^{2} \partial_{i} u \nabla w_{n}^{i}\right) d x=0 .
$$

Theorems 3.4 and 3.6 are consequences of the following lemma:

Lemma 3.7. Assume that there exist $x_{0} \in \Omega, \delta>0, w^{1}, w^{2}$ in $H^{1}\left(B\left(x_{0}, \delta\right)\right) \cap C^{1}\left(B\left(x_{0}, \delta\right)\right)$ and two sequences $w_{n}^{1}, w_{n}^{2}$ in $H^{1}\left(B\left(x_{0}, \delta\right)\right), n \in \mathbb{N}$, which satisfy $(3.7)$.

Then, there exists $\varepsilon \in(0, \delta)$, a nonnegative bounded Radon measure $\mu$ on $B\left(x_{0}, \delta\right)$ and a nonnegative matrix-valued function $A$ in $L_{\mu}^{\infty}\left(B\left(x_{0}, \varepsilon\right)\right)^{2 \times 2}$, such that for any open subset $\omega$ of $\Omega$, with $B\left(x_{0}, \varepsilon\right) \cap \omega \neq \varnothing$, the sequence $F_{n}(\cdot, \omega)$ defined by (3.6) $\Gamma$-converges for the strong topology of $L^{2}(\omega)$ (up to a subsequence of $n$, still denoted by $n$, which depends on $\omega$ ) to the Dirichlet form $F(\cdot, \omega)$ which satisfies the following properties:

(i) The space $\left.C_{c}^{1}\left(B\left(x_{0}, \varepsilon\right) \cap \omega\right)\right)$ is contained in $D(F(\cdot, \omega))$, and

$$
F(u, \omega)=\int_{B\left(x_{0}, \varepsilon\right) \cap \omega} A \nabla u \cdot \nabla u d \mu, \quad \forall u \in C_{c}^{1}\left(B\left(x_{0}, \varepsilon\right) \cap \omega\right) .
$$

(ii) For any $u \in D(F(., \omega)) \cap C_{0}^{1}(\omega)$, and any sequence $u_{n}$ in $H_{0}^{1}(\omega)$, which strongly converges to $u$ in $L^{2}(\omega)$ and such that $F_{n}\left(u_{n}, \omega\right)$ tends to $F(u, \omega)$, the sequence $A_{n} \nabla u_{n}$. $\nabla u_{n}$ converges to $A \nabla u \cdot \nabla u d \mu$ in the weak $*$ sense of the measures in $B\left(x_{0}, \varepsilon\right) \cap \omega$.

Theorem 3.4 refers to the case where $D(F)$ contains $C_{c}^{1}(\Omega)$. In the general case we obtain a characterization of the $\Gamma$-limit of $F_{n}(\cdot, \omega)(3.6)$ but not explicit like the representation formula (3.9). In fact, we have the following abstract result:

Theorem 3.8. Let $\mathcal{R}$ be the equivalence relation defined in $\bar{\Omega}$ by

$$
x \mathcal{R} y \Leftrightarrow u(x)=u(y), \quad \forall u \in D(F) \cap C_{0}(\Omega),
$$

and note that any function $u \in D(F) \cap C_{0}(\Omega)$ defines a function in $\bar{\Omega} / \mathcal{R}$, still denoted by $u$. The set $\bar{\Omega} / \mathcal{R}$ is endowed with the smallest topology $\mathcal{T}$ such that the functions in $D(F) \cap$ $C_{0}(\Omega)$ are continuous for $\mathcal{T}$. Then, $\bar{\Omega} / \mathcal{R}$ is a Hausdorff, separable and compact topological space.

We denote by $\Omega^{*}$ the set $\bar{\Omega} / \mathcal{R}$ without the class containing the elements of $\partial \Omega$. Then, $\Omega^{*}$ is locally compact and the set $D(F) \cap C_{0}(\Omega)$ is a dense separating subalgebra of $C_{0}\left(\Omega^{*}\right)$, which allows us to define a bounded Radon measure $m$ on $\Omega^{*}$ by

$$
\int_{\Omega^{*}} u d m=\int_{\Omega} u d x, \quad \forall u \in D(F) \cap C_{0}(\Omega) .
$$

Then, the restriction of the $\Gamma$-limit $F$ to $D(F) \cap C_{0}(\Omega)$ is a closable Markovian form in $L_{m}^{2}\left(\Omega^{*}\right)$, which is strongly local. Its closure $F^{*}$ is a strongly local Dirichlet form. 
Remark 3.9. By Theorem 3.8 the Dirichlet form $F^{*}$ is a diffusion. Denote by $\mathcal{M}^{*}$ the space of the Radon measures on $\Omega^{*}$. Then, following Mosco [18] there exists a bilinear form $\nu: D\left(F^{*}\right) \times D\left(F^{*}\right) \longrightarrow \mathcal{M}^{*}$, such that

$$
F^{*}(u)=\int_{\Omega^{*}} d \nu(u, u), \quad \forall u \in D\left(F^{*}\right) .
$$

For any $u \in D\left(F^{*}\right)$, the measure $\nu(u, u)$ is nonnegative and depends locally on $u$. That is, if $u_{1}, u_{2} \in D\left(F^{*}\right)$ agree in an open subset $G$ of $\Omega^{*}$, then $\nu\left(u_{1}, u_{1}\right)=\nu\left(u_{2}, u_{2}\right)$ on $G$. Moreover, the measure $\nu$ satisfies several properties which are detailed in [18], such as the Leibnitz rule, the chain rule and the truncation principle.

On the other hand, by the Stone-Weierstrass theorem, $D(F) \cap C_{0}(\Omega)$ is dense in $C_{0}(\Omega)$ if and only if, for any $x \in \Omega$, the class of $x$ by the relation $\mathcal{R}$ reduces to $\{x\}$. In this case, the sets $\Omega$ and $\Omega^{*}$ may be identified. However, even under the assumption $\Omega \equiv \Omega^{*}$, we cannot express $F^{*}$ more precisely, since we do not know the exact composition of the domain $D(F)$. We also refer to [18] (p. 192) for an explicit treatment of the diffusion part of a Dirichlet form.

\subsection{Proof of the results}

Proof of Lemma 3.7. First, since by Properties $3.2 a$ ) the sequence $F_{n}^{*}$ defined by

$$
F_{n}^{*}\left(u, B\left(x_{0}, \delta\right)\right):=\left\{\begin{array}{cl}
\int_{B\left(x_{0}, \delta\right)} A_{n} \nabla u \cdot \nabla u d x & \text { if } u \in H^{1}\left(B\left(x_{0}, \delta\right)\right) \\
+\infty & \text { if } u \in L^{2}\left(B\left(x_{0}, \delta\right)\right) \backslash H^{1}\left(B\left(x_{0}, \delta\right)\right),
\end{array}\right.
$$

$\Gamma$-converges, up a subsequence, for the strong topology of $L^{2}\left(B\left(x_{0}, \delta\right)\right)$, we may choose $w_{n}^{1}$, $w_{n}^{2}$ which satisfy $(3.7)$ with limits $w^{1}, w^{2}$, as recovery sequences for $F_{n}^{*}$. Then, thanks to Properties $3.2 c$ ) the sequence $w_{n}^{i}$, for $i \in\{1,2\}$, satisfies

$$
\left\{\begin{array}{l}
\lim _{n \rightarrow+\infty} \int_{B\left(x_{0}, \delta\right)} A_{n} \nabla w_{n}^{i} \cdot \nabla v_{n} d x=0, \quad \forall v_{n} \in H^{1}\left(B\left(x_{0}, \delta\right)\right), \\
v_{n} \rightarrow 0 \text { strongly in } L^{2}\left(B\left(x_{0}, \delta\right)\right), \quad A_{n} \nabla v_{n} \cdot \nabla v_{n} \text { bounded in } L^{1}\left(B\left(x_{0}, \delta\right)\right),
\end{array}\right.
$$

and by Theorem $2.1 w_{n}^{i}$ strongly converges to $w^{i}$ in $L_{\text {loc }}^{\infty}\left(B\left(x_{0}, \delta\right)\right)$.

On the other hand, we define the Radon measure $\mu$ on $B\left(x_{0}, \delta\right)$ and the matrix-valued $A$ in $L_{\mu}^{\infty}(\Omega)^{2 \times 2}$ by the following weak * convergences which hold true up to a subsequence,

$$
\begin{cases}A_{n} \nabla w_{n}^{1} \cdot \nabla w_{n}^{1}+A_{n} \nabla w_{n}^{2} \cdot \nabla w_{n}^{2} \rightarrow \mu & \text { in } \mathcal{M}\left(\bar{B}\left(x_{0}, \delta\right)\right) *, \\ A_{n} \nabla w_{n}^{i} \cdot \nabla w_{n}^{j} d x \rightarrow A \nabla w^{i} \cdot \nabla w^{j} d \mu & \text { in } \mathcal{M}\left(\bar{B}\left(x_{0}, \delta\right)\right) *, \quad i, j \in\{1,2\} .\end{cases}
$$

Since $\nabla w^{1}\left(x_{0}\right), \nabla w^{2}\left(x_{0}\right)$ are linearly independent, there exists $\varepsilon \in(0, \delta)$ and an open subset $O$ of $\mathbb{R}^{2}$, such that $\nabla w^{1}, \nabla w^{2}$ are linearly independent in $B\left(x_{0}, \varepsilon\right)$ and the function $w:=\left(w_{1}, w_{2}\right)$ is one-to-one from $B\left(x_{0}, \varepsilon\right)$ onto $O$, with $C^{1}$ inverse.

Proof of $(i)$. Let us consider an open subset $\omega$ of $\Omega$, with $\omega \cap B\left(x_{0}, \varepsilon\right) \neq \varnothing$, and a new subsequence of $n$ (which depends on $\omega$ ), still denoted by $n$, such that $F_{n}(\cdot, \omega) \Gamma$-converges to $F(\cdot, \omega)$ for the strong topology of $L^{2}(\omega)$.

Then, for a given function $u \in C_{c}^{1}\left(B\left(x_{0}, \varepsilon\right) \cap \omega\right)$, we may define the function

$$
R:=u\left(w^{-1}\right) \in C_{c}^{1}\left(w\left(B\left(x_{0}, \varepsilon\right) \cap \omega\right)\right),
$$

so that $u=R(w)$ in $B\left(x_{0}, \varepsilon\right) \cap \omega$. Set $w_{n}:=\left(w_{n}^{1}, w_{n}^{2}\right)$. Due to the uniform convergence of $w_{n}^{i}$, and to the compactness of $\operatorname{supp}(R)$ in $w\left(B\left(x_{0}, \varepsilon\right) \cap \omega\right)$, the function $R\left(w_{n}\right)$ belongs 
to $H_{0}^{1}\left(B\left(x_{0}, \varepsilon\right) \cap \omega\right)$. Thus, denoting by $u_{n}$ the extension of $R\left(w_{n}\right)$ by zero outside of $B\left(x_{0}, \varepsilon\right) \cap \omega, u_{n}$ is a sequence in $H_{0}^{1}(\omega)$, which strongly converges to $u$ in $L^{\infty}(\omega)$. On the other hand, using that

$$
F_{n}\left(u_{n}, \omega\right)=\sum_{i, j=1}^{2} \int_{B\left(x_{0}, \varepsilon\right) \cap \omega} A_{n}\left(\partial_{i} R\left(w_{n}\right) \nabla w_{n}^{i}\right) \cdot\left(\partial_{j} R\left(w_{n}\right) \nabla w_{n}^{j}\right) d x
$$

is bounded, we deduce that $u$ belongs to $D(F(., \omega))$. Moreover, formula (3.14) combined with (3.13) and the uniform convergence of $\partial_{i} R\left(w_{n}\right)$, yields

$$
\lim _{n \rightarrow+\infty} F_{n}\left(u_{n}, \omega\right)=\int_{B\left(x_{0}, \varepsilon\right) \cap \omega} A \nabla u \cdot \nabla u d \mu .
$$

Therefore, it remains to prove that

$$
F(u, \omega)=\lim _{n \rightarrow+\infty} F_{n}\left(u_{n}, \omega\right),
$$

in order to obtain the desired formula (3.11).

First, note that Remark 2.2 implies that the $\Gamma$-limit of $F_{n}(\cdot, \omega)$ for the strong topology of $L^{2}(\omega)$ agrees with the $\Gamma$-limit for the strong topology of $L^{\infty}(\omega)$ over $H_{0}^{1}(\omega) \cap C_{0}(\omega)$. Therefore, thanks to Properties $3.2 c$ ), to prove (3.15) it is enough to check that for any sequence $v_{n}$ in $H_{0}^{1}(\omega)$, which strongly converges to zero in $L^{\infty}(\omega)$ and such that $A_{n} \nabla v_{n}$. $\nabla v_{n}$ is bounded in $L^{1}(\omega)$,

$$
\lim _{n \rightarrow+\infty} \int_{\omega} A_{n} \nabla u_{n} \cdot \nabla v_{n} d x=\lim _{n \rightarrow+\infty} \sum_{i=1}^{2} \int_{B\left(x_{0}, \varepsilon\right) \cap \omega} A_{n}\left(\partial_{i} R\left(w_{n}\right) \nabla w_{n}^{i}\right) \cdot \nabla v_{n} d x=0 .
$$

For such a sequence $v_{n}$, we consider, for $\rho>0, R_{\rho} \in C_{c}^{2}\left(w\left(B\left(x_{0}, \varepsilon\right) \cap \omega\right)\right)$ such that

$$
\left\|R-R_{\rho}\right\|_{C^{1}\left(w\left(B\left(x_{0}, \varepsilon\right) \cap \omega\right)\right)}<\rho .
$$

For $i \in\{1,2\}$, we start from the equality

$$
\begin{aligned}
& \int_{B\left(x_{0}, \varepsilon\right) \cap \omega} A_{n}\left(\partial_{i} R\left(w_{n}\right) \nabla w_{n}^{i}\right) \cdot \nabla v_{n} d x=\int_{B\left(x_{0}, \varepsilon\right) \cap \omega} A_{n}\left(\partial_{i} R_{\rho}\left(w_{n}\right) \nabla w_{n}^{i}\right) \cdot \nabla v_{n} d x \\
& +\int_{B\left(x_{0}, \varepsilon\right) \cap \omega} A_{n}\left(\left(\partial_{i} R\left(w_{n}\right)-\partial_{i} R_{\rho}\left(w_{n}\right)\right) \nabla w_{n}^{i}\right) \cdot \nabla v_{n} d x .
\end{aligned}
$$

The second term of the right-hand side of (3.17) clearly satisfies

$$
\limsup _{n \rightarrow+\infty}\left|\int_{B\left(x_{0}, \varepsilon\right) \cap \omega} A_{n}\left(\partial_{i}\left(R\left(w_{n}\right)-R_{\rho}\left(w_{n}\right)\right) \nabla w_{n}^{i}\right) \cdot \nabla v_{n} d x\right| \leq C \rho .
$$

For the first term, defining $r_{n} \in H_{0}^{1}(\Omega)$ as the extension of $\partial_{i} R_{\rho}\left(w_{n}\right)$ by zero outside $B\left(x_{0}, \varepsilon\right) \cap \omega$, we have

$$
\begin{aligned}
& \int_{B\left(x_{0}, \varepsilon\right) \cap \omega} A_{n}\left(\partial_{i} R_{\rho}\left(w_{n}\right) \nabla w_{n}^{i}\right) \cdot \nabla v_{n} d x \\
& =\int_{\omega} A_{n} \nabla w_{n}^{i} \cdot \nabla\left(r_{n} v_{n}\right) d x-\int_{B\left(x_{0}, \varepsilon\right) \cap \omega} A_{n} \nabla w_{n}^{i} \cdot \nabla\left(\partial_{i} R_{\rho}\left(w_{n}\right)\right) v_{n} d x
\end{aligned}
$$

where by (3.12) and the uniform convergence of $v_{n}$ to zero, the right-hand side tends to zero. Therefore, we obtain, for any $\rho>0$ and $i \in\{1,2\}$,

$$
\limsup _{n \rightarrow+\infty}\left|\int_{\Omega} A_{n}\left(\partial_{i} R\left(w_{n}\right) \nabla w_{n}^{i}\right) \cdot \nabla v_{n} d x\right| \leq C \rho
$$


which proves (3.16) and thus (3.15).

Proof of $(i i)$. Let us consider $u \in D(F(., \omega)) \cap C_{0}^{1}(\omega)$ and a sequence $u_{n}$ in $H_{0}^{1}(\omega)$, which strongly converges to $u$ in $L^{2}(\omega)$ and such that $F_{n}\left(u_{n}, \omega\right)$ tends to $F(u, \omega)$. Thanks to Remark 2.2 there exists another sequence $\hat{u}_{n}$ satisfying the same properties but which also strongly converges to $u$ in $L^{\infty}(\omega)$. Since $u_{n}$ and $\hat{u}_{n}$ are recovery sequences $(3.2)$ for $F_{n}(\cdot, \omega)$, with the same limit $u$, Properties $3.2 c$ ) implies that

$$
\lim _{n \rightarrow+\infty} \int_{\omega} A_{n} \nabla\left(u_{n}-\hat{u}_{n}\right) \cdot \nabla\left(u_{n}-\hat{u}_{n}\right) d x=0,
$$

hence the weak $*$ limits of $A_{n} \nabla u_{n} \cdot \nabla u_{n}$ and $A_{n} \nabla \hat{u}_{n} \cdot \nabla \hat{u}_{n}$ in the sense of the measures in $\omega$ coincide. So, replacing $u_{n}$ by $\hat{u}_{n}$ we can assume that $u_{n}$ strongly converges to $u$ in $L^{\infty}(\omega)$.

For $\varphi \in C_{c}^{1}\left(B\left(x_{0}, \varepsilon\right) \cap \omega\right)$, we take $v \in C_{c}^{1}\left(B\left(x_{0}, \varepsilon\right)\right)$ such that $v=u$ in $\operatorname{supp}(\varphi)$. Then, we consider two sequences $\varphi_{n}, v_{n}$ in $H_{0}^{1}(\omega)$, which strongly converge in $L^{\infty}(\omega)$ respectively to $\varphi, v$ and satisfy

$$
\lim _{n \rightarrow+\infty} F_{n}\left(\varphi_{n}, \omega\right)=F(\varphi, \omega) \text { and } \lim _{n \rightarrow+\infty} F_{n}\left(v_{n}, \omega\right)=F(v, \omega) .
$$

Using the second result of Theorem 2.1 we may also choose $\varphi_{n}$ such that $\operatorname{supp}\left(\varphi_{n}\right)$ is contained in $\operatorname{supp}(\varphi)$. We have

$$
\begin{aligned}
& \int_{\omega} A_{n} \nabla u_{n} \cdot \nabla u_{n} \varphi d x=\int_{\omega} A_{n} \nabla u_{n} \cdot \nabla u_{n}\left(\varphi-\varphi_{n}\right) d x \\
& +\int_{\omega} A_{n} \nabla\left(u_{n}-v_{n}\right) \cdot \nabla u_{n} \varphi_{n} d x+\int_{\omega} A_{n} \nabla v_{n} \cdot \nabla\left(u_{n}-v_{n}\right) \varphi_{n} d x \\
& +\int_{\omega} A_{n} \nabla v_{n} \cdot \nabla\left(v_{n} \varphi_{n}\right) d x-\frac{1}{2} \int_{\omega} A_{n} \nabla v_{n}^{2} \cdot \nabla \varphi_{n} d x .
\end{aligned}
$$

The first term of the right-hand side of (3.19) tends to zero thanks to the uniform convergence of $\varphi_{n}$. For the second term, we use

$$
\begin{aligned}
\int_{\omega} A_{n} \nabla\left(u_{n}-v_{n}\right) \cdot \nabla u_{n} \varphi_{n} d x & =\int_{\omega} A_{n} \nabla\left(\left(u_{n}-v_{n}\right) \varphi_{n}\right) \cdot \nabla u_{n} d x \\
& -\int_{\omega} A_{n} \nabla \varphi_{n} \cdot \nabla u_{n}\left(u_{n}-v_{n}\right) d x .
\end{aligned}
$$

Combining the convergence of $F_{n}\left(u_{n}, \omega\right)$ to $F(u, \omega)$, the strong convergence of $\left(u_{n}-v_{n}\right) \varphi_{n}$ to zero in $L^{2}(\omega)$ and the boundedness of $A_{n} \nabla\left(\left(u_{n}-v_{n}\right) \varphi_{n}\right) \cdot \nabla\left(\left(u_{n}-v_{n}\right) \varphi_{n}\right)$ in $L^{1}(\omega)$, we obtain by (3.3) that the first term of the right-hand side of (3.20) tends to zero. The second term also tends to zero thanks to the uniform convergence of $u_{n}-v_{n}$ to zero in $\operatorname{supp}(\varphi) \supset \operatorname{supp}\left(\varphi_{n}\right)$. Therefore, the second term of the right-hand side of (3.19) tends to zero. Similarly, the third term of the right-hand side of (3.19) converges to zero.

For the fourth term of the right-hand side of (3.19) we apply Properties $3.2 c$ ) to $v_{n}$ which is a recovery sequence by (3.18). Then, since $v$ and $\varphi$ have support in $B\left(x_{0}, \varepsilon\right) \cap \omega$ and $v=u$ in $\operatorname{supp} \varphi$, the part $(i)$ of Lemma 3.7 implies that

$$
\lim _{n \rightarrow+\infty} \int_{\omega} A_{n} \nabla v_{n} \cdot \nabla\left(v_{n} \varphi_{n}\right) d x=\int_{\omega} A \nabla u \cdot \nabla(u \varphi) d \mu .
$$

Similarly, since $\varphi_{n}$ is a recovery sequence by (3.18), we have

$$
\lim _{n \rightarrow+\infty} \int_{\omega} A_{n} \nabla v_{n}^{2} \cdot \nabla \varphi_{n} d x=\int_{\omega} A \nabla u^{2} \cdot \nabla \varphi d \mu
$$


Finally, passing to the limit in the right-hand side of (3.19) thanks to the previous convergences, yields

$$
\lim _{n \rightarrow+\infty} \int_{\omega} A_{n} \nabla u_{n} \cdot \nabla u_{n} \varphi d x=\int_{\omega} A \nabla u \cdot \nabla u \varphi d \mu, \quad \forall \varphi \in C_{c}^{1}\left(B\left(x_{0}, \varepsilon\right) \cap \omega\right),
$$

which concludes the proof of Lemma 3.7.

Proof of Theorem 3.4. If $C_{c}^{1}(\Omega)$ is contained in $D(F)$, then it is clear that for any $x_{0} \in \Omega$, there exists $\delta>0$, two functions $w^{1}, w^{2}$ in $C^{1}\left(B\left(x_{0}, \delta\right)\right)$ and two sequences $w_{n}^{1}, w_{n}^{2}$ in $H^{1}\left(B\left(x_{0}, \delta\right)\right)$ which satisfy $(3.7)$.

Inversely, if condition (3.7) is satisfied, Lemma 3.7 proves that for any $x_{0} \in \Omega$, there exist $\varepsilon>0$, a nonnegative bounded Radon measure $\hat{\mu}$ and a nonnegative matrix-valued function $\hat{A}$ in $L_{\hat{\mu}}^{\infty}\left(B\left(x_{0}, \varepsilon\right)\right)^{2 \times 2}$ such that (3.11) holds true for any open subset $\omega$ of $\Omega$ and for the $\Gamma$-limit $F(\cdot, \omega)$ of any convergent $\Gamma$-subsequence of $F_{n}(\cdot, \omega)$ in $L^{2}(\omega)$, with $C_{c}^{1}\left(B\left(x_{0}, \varepsilon\right)\right) \subset D(F(\cdot, \omega))$. ¿From the covering of $\Omega$ by the disks $B\left(x_{0}, \varepsilon\right)$, we can deduce the existence of $x_{i} \in \Omega, \varepsilon_{i}>0$ and $\varphi_{i} \in C_{c}^{\infty}\left(B\left(x_{i}, \varepsilon_{i}\right)\right), i \in \mathbb{N}^{*}$, such that any compact subset of $\Omega$ only intersects a finite number of $B\left(x_{i}, \varepsilon_{i}\right)$ and $\sum_{i \in \mathbb{N}^{*}} \varphi_{i}(x)=1$ in $\Omega$. Then, considering the Radon measure $\mu_{i}$ and the matrix-valued function $A_{i}$ associated with each disk $B\left(x_{i}, \varepsilon_{i}\right)$, for $i \in \mathbb{N}^{*}$, according to the procedure of Lemma 3.7 combined with a diagonal extraction, we define the measure $\mu$ by

$$
\mu(B):=\sum_{i=1}^{\infty} \mu_{i}\left(B\left(x_{i}, \varepsilon_{i}\right) \cap B\right), \quad \forall B \text { Borel subset of } \Omega,
$$

and, using the Radon-Nikodym theorem we define the matrix-valued measure $A d \mu$ by

$$
\int_{B} A d \mu:=\sum_{i \in \mathbb{N}^{*}} \int_{B\left(x_{i}, \varepsilon_{i}\right) \cap B} A^{i} \varphi_{i} d \mu_{i} \quad \forall B \text { Borel set, with } \bar{B} \subset \Omega .
$$

Now, let us consider an open subset $\omega$ of $\Omega$ and $u \in C_{c}^{1}(\omega)$. We have

$$
u=\sum_{i=1}^{\infty} u \varphi_{i}
$$

where the sum carries on a finite set of indexes $i$. Since by Lemma 3.7 each function $u \varphi_{i}$ is in $D(F(\cdot, \omega))$, the function $u$ also belongs to $D(F(\cdot, \omega))$. Let $u_{n}$ be a sequence in $H_{0}^{1}(\omega)$, which strongly converges to $u$ in $L^{2}(\omega)$ and such that $F_{n}\left(u_{n}, \omega\right)$ tends to $F(u, \omega)$. By the part (ii) of Lemma 3.7 the sequence $A_{n} \nabla u_{n} \cdot \nabla u_{n}$ converges to $A^{i} \nabla u \cdot \nabla u d \mu_{i}$ in the weak * sense of the measures in $B\left(x_{i}, \varepsilon_{i}\right) \cap \omega$, for each $i \in \mathbb{N}^{*}$. Then, since for any $\varphi \in C_{c}^{1}(\omega)$, $\operatorname{supp}(\varphi) \cap \operatorname{supp}\left(\varphi_{i}\right) \neq \varnothing$ only for a finite set of indexes $i$, we have

$$
\begin{aligned}
& \lim _{n \rightarrow+\infty} \int_{\omega} A_{n} \nabla u_{n} \cdot \nabla u_{n} \varphi d x=\lim _{n \rightarrow+\infty} \sum_{i=1}^{\infty} \int_{\omega} \varphi_{i} A_{n} \nabla u_{n} \cdot \nabla u_{n} \varphi d x \\
& =\sum_{i=1}^{\infty} \int_{\omega} \varphi_{i} A^{i} \nabla u \cdot \nabla u \varphi d \mu_{i}=\int_{\omega} A \nabla u \cdot \nabla u \varphi d \mu .
\end{aligned}
$$

This proves that $A_{n} \nabla u_{n} \cdot \nabla u_{n}$ weakly converges to $A \nabla u \cdot \nabla u d \mu$ in $\mathcal{N}(\omega) *$. Thanks to second result of Theorem 2.1 we can choose $u_{n}$ such that $\operatorname{supp}\left(u_{n}\right) \subset \operatorname{supp}(u)$. Then, taking in $(3.21) \varphi \in C_{c}^{1}(\omega)$ such that $\varphi=1$ in $\operatorname{supp}(u)$, we get

$$
\begin{aligned}
& F(u, \omega)=\lim _{n \rightarrow+\infty} F_{n}\left(u_{n}, \omega\right)=\lim _{n \rightarrow+\infty} \int_{\omega} A_{n} \nabla u_{n} \cdot \nabla u_{n} d x=\lim _{n \rightarrow+\infty} \int_{\omega} A_{n} \nabla u_{n} \cdot \nabla u_{n} \varphi d x \\
& =\int_{\omega} A \nabla u \cdot \nabla u \varphi d \mu=\int_{\omega} A \nabla u \cdot \nabla u \varphi d \mu=\int_{\omega} A \nabla u \cdot \nabla u d \mu,
\end{aligned}
$$


which proves (3.9). Moreover, by (2.1) the sequence $u_{n}$ converges weakly to $u$ in $H_{0}^{1}(\Omega)$, and for any $u \in C_{c}^{1}(\omega)$, we have

$$
\alpha \int_{\Omega}|\nabla u|^{2} d x \leq \alpha \liminf _{n \rightarrow+\infty} \int_{\Omega}\left|\nabla u_{n}\right|^{2} d x \leq \lim _{n \rightarrow+\infty} F_{n}\left(u_{n}, \omega\right)=F(u, \omega)=\int_{\Omega} A \nabla u \cdot \nabla u d \mu .
$$

This implies (3.8) (see e.g. [13] Lemma 22.5 p. 234) and concludes the proof.

Proof of Theorem 3.6. Similarly to the proof of Theorem 3.4 we consider $\varphi^{j} \in C_{c}^{1}(\Omega)$, for $j \in \mathbb{N}$, which gives a partition of the unity associated with a locally finite covering of $\Omega$. For any $i \in\{1,2\}$ and any $j \in \mathbb{N}$, we consider $z_{n}^{i, j} \in H_{0}^{1}(\Omega)$, with $z_{n}^{i, j}=0$ q.e. outside $\operatorname{supp}\left(\varphi^{j}\right)$, such that $z_{n}^{i, j}$ strongly converges to $x_{i} \varphi^{j}$ in $L^{\infty}(\Omega)$ and $F_{n}\left(z_{n}^{i, j}\right)$ tends to $F\left(x_{i} \varphi^{j}\right)$. Then, for $i \in\{1,2\}$, we define

$$
w_{n}^{i}:=\sum_{j \in J_{n}} z_{n}^{i, j}, \quad \text { where } \quad J_{n}:=\left\{j \in \mathbb{N}: \operatorname{supp}\left(\varphi_{j}\right) \cap\left\{x \in \Omega: \operatorname{dist}(x, \partial \Omega)<\frac{1}{n}\right\}=\varnothing\right\} .
$$

The sequences $w_{n}^{i}$ clearly satisfy the conditions (3.7) of Theorem 3.6.

Now, consider an open subset $\omega$ of $\Omega$, a function $u \in C_{c}^{1}(\omega)$, and assume that $F_{n}(\cdot, \omega)$ $\Gamma$-converges to $F(\cdot, \omega)$ for the strong topology of $L^{2}(\omega)$. Since the sequence $w_{n}:=\left(w_{n}^{1}, w_{n}^{2}\right)$ strongly converges to the identity in $L_{\text {loc }}^{\infty}(\Omega)$, the argument used in the proof of Lemma 3.7 shows that the sequence $F_{n}\left(u\left(w_{n}\right), \omega\right)$ converges to $F(u, \omega)$. So, for any recovery sequence $u_{n}$ in $H_{0}^{1}(\omega)$, which strongly converges to $u$ in $L^{2}(\omega)$ and such that $F_{n}\left(u_{n}, \omega\right)$ tends to $F(u, \omega)$, Properties $3.2 c$ ) implies that

$$
\lim _{n \rightarrow+\infty} \int_{\omega} A_{n} \nabla\left(u_{n}-u\left(w_{n}\right)\right) \cdot \nabla\left(u_{n}-u\left(w_{n}\right)\right) d x=0 .
$$

On the other hand, since $w_{n}$ uniformly converges to the identity and $u \in C_{c}^{1}(\omega)$, we also have

$$
\begin{aligned}
& \lim _{n \rightarrow+\infty} \int_{\omega} A_{n}\left(\nabla\left[u\left(w_{n}\right)\right]-\sum_{i=1}^{2} \partial_{i} u \nabla w_{n}^{i}\right) \cdot\left(\nabla\left[u\left(w_{n}\right)\right]-\sum_{i=1}^{2} \partial_{i} u \nabla w_{n}^{i}\right) d x \\
& =\lim _{n \rightarrow+\infty} \sum_{i, k=1}^{2} \int_{\omega} A_{n} \nabla w_{n}^{i} \cdot \nabla w_{n}^{k}\left(\partial_{i} u\left(w_{n}\right)-\partial_{i} u\right)\left(\partial_{k} u\left(w_{n}\right)-\partial_{k} u\right) d x=0,
\end{aligned}
$$

which combined with (3.22) yields the desired limit (3.10).

Proof of Theorem 3.8. For $x \in \bar{\Omega}$, we denote by $[x]$ its class in $\bar{\Omega} / \mathcal{R}$. The class containing the elements of $\partial \Omega$ (note that all the elements of $\partial \Omega$ are in relation by $\mathcal{R}$ ) is denoted by $[\partial]$. A basis for the topology $\mathcal{T}$ is given by the subsets of $\bar{\Omega} / \mathcal{R}$ of the form

$$
\bigcap_{i=1}^{n} u_{i}^{-1}\left(\left(s_{i}-\varepsilon_{i}, s_{i}+\varepsilon_{i}\right)\right),
$$

with $u_{1}, \ldots, u_{n} \in D(F) \cap C_{0}(\Omega), s_{1}, \ldots, s_{n} \in \mathbb{R}, \varepsilon_{1}, \ldots, \varepsilon_{n}>0$ and $n \in \mathbb{N}^{*}$. Then, by definition of $\mathcal{R}$, for any $x, y \in \bar{\Omega}$ with $[x] \neq[y]$, there exists $u \in D(F) \cap C_{0}(\Omega)$ such that $u(x) \neq u(y)$. Therefore, $\mathcal{T}$ is Hausdorff.

Let $x \in \bar{\Omega}$ and let $V$ be a neighbourhood of $[x]$ of type (3.23). By the density of $\mathbb{Q}^{2}$ in $\mathbb{R}^{2}$ and the continuity of the functions $u_{i}$ in (3.23), there exists $z \in \mathbb{Q}^{2} \cap \bar{\Omega}$ such that $[z] \in V$. Therefore, the classes of the elements in $\mathbb{Q}^{2} \cap \bar{\Omega}$ are dense in $\bar{\Omega} / \mathcal{R}$, which implies the separability of $\mathcal{T}$.

By the definition of $\mathcal{T}$, for any open set $\mathcal{O}$ of $\bar{\Omega} / \mathcal{R}$, the set $\{x \in \Omega:[x] \in \mathcal{O}\}$ is an open subset of $\bar{\Omega}$. By considering the complementary the same property holds true for 
the closed sets. Using the compactness of $\bar{\Omega}$, the characterization of the open sets implies that $\bar{\Omega} / \mathcal{R}$ is compact and thus, the set $\Omega^{*}:=\bar{\Omega} / \mathcal{R} \backslash\{[\partial]\}$ is locally compact.

Using that the $\Gamma$-limit $F$ of $F_{n}(3.6)$ is closed for the strong topology of $L^{2}(\Omega)$ and the definition of the measure $m$, the restriction of $F$ to $D(F) \cap C_{0}(\Omega)$ is a closable form in $L_{m}^{2}\left(\Omega^{*}\right)$, whose closure is denoted by $F^{*}$. Let us prove that $F^{*}$ is a regular Dirichlet form according to Definition 3.3.

Set $H(s):=(s \vee 0) \wedge 1$, for $s \in \mathbb{R}$. Then, for any $u \in D(F)$ and any recovery sequence $u_{n} \in H_{0}^{1}(\Omega)$ associated with $u$ and $F_{n}$ by (3.2), we have

$$
F(H(u)) \leq \liminf _{n \rightarrow+\infty} F_{n}\left(H\left(u_{n}\right)\right) \leq \lim F_{n}\left(u_{n}\right)=F(u) .
$$

In particular, this holds for any $u \in D(F) \cap C_{0}(\Omega)$, hence the restriction of $F$ to $D(F) \cap$ $C_{0}(\Omega)$ is Markovian.

Since $D(F) \cap C_{0}(\Omega)$ is an algebra which separates points, the Stone-Weierstrass theorem shows that the functions of the form $u+c$, with $u \in D(F) \cap C_{0}(\Omega)$ and $c \in \mathbb{R}$, are dense in $C(\bar{\Omega} / \mathcal{R})$. Now, consider $v \in C_{c}\left(\Omega^{*}\right), v_{n} \in D(F) \cap C_{0}(\Omega)$ and $c_{n} \in \mathbb{R}$, such that $v_{n}+c_{n}$ converges to $v$ in $C(\bar{\Omega} / \mathcal{R})$. Since $v$ and $v_{n}$ vanish in $[\partial]$, the sequence $c_{n}$ converges to zero and thus $v_{n}$ converges to $v$ in $C(\bar{\Omega} / \mathcal{R})$. This proves that $D(F) \cap C_{0}(\Omega)$ is dense in $C_{0}\left(\Omega^{*}\right)$, which implies that $F^{*}$ is regular. Therefore, $F^{*}$ is a regular Dirichlet form.

It remains to prove that $F^{*}$ is strongly local, i.e., the polar form $\Phi$ of $F$ satisfies (3.4). Let $u, v \in D(F) \cap C_{0}(\Omega)$ and $c \in \mathbb{R}$, such that $u=c$ constant in $\operatorname{supp}(v)$. First, we assume that $c \geq 0$. Taking into account Remark 2.2, we consider two recovery sequences $u_{n}, v_{n}$ which strongly converge respectively to $u, v$ in $L^{\infty}(\Omega)$ and such that $F_{n}\left(u_{n}\right), F_{n}\left(v_{n}\right)$ tend respectively to $F(u), F(v)$. We also choose $v_{n}$ such that $\operatorname{supp}\left(v_{n}\right) \subset \operatorname{supp}(v)$. Let $H_{\varepsilon}$, for $\varepsilon>0$, be the function defined in $\mathbb{R}$ by

$$
H_{\varepsilon}(s):= \begin{cases}\frac{c}{c-\varepsilon} s & \text { if } s<c-\varepsilon \\ c & \text { if } c-\varepsilon \leq s \leq c+\varepsilon \quad \text { if } \varepsilon<c, \\ s+\varepsilon & \text { if } s>c+\varepsilon\end{cases}
$$

(note that $H_{\varepsilon}(0)=0$ ), and $H_{\varepsilon}(s):=(s-\varepsilon \operatorname{sgn}(s)) \chi_{\{|s|>\varepsilon\}}$ if $c=0$. The sequence $\varepsilon_{n}:=\left\|u_{n}-u\right\|_{L^{\infty}(\Omega)}$ converges to zero. Then, the sequence $H_{\varepsilon_{n}}\left(u_{n}\right)$ satisfies the same properties than $u_{n}$, but we also have $H_{\varepsilon_{n}}\left(u_{n}\right)=c$ in $\operatorname{supp}(v) \supset \operatorname{supp}\left(v_{n}\right)$, for $\varepsilon_{n}$ small enough. Therefore, the Properties $3.2 c$ ) of the recovery sequence $v_{n}$ yields

$$
\Phi(u, v)=\lim _{n \rightarrow+\infty} \int_{\Omega} A_{n} \nabla\left(H_{\varepsilon_{n}}\left(u_{n}\right)\right) \cdot \nabla v_{n} d x=0 .
$$

In the case $c<0$, we simply use the equality $\Phi(u, v)=-\Phi(-u, v)=0$.

\section{Application to the periodic case}

\subsection{Statement of the results}

In this section we consider a sequence $B_{n}$ of symmetric matrix-valued functions in $L^{\infty}\left(\mathbb{R}^{2}\right)^{2 \times 2}$, which satisfies the following assumptions:

$B_{n}$ is $Y$-periodic, where $Y:=(0,1)^{2}$, i.e.,

$$
\forall n \in \mathbb{N}, \forall \kappa \in \mathbb{Z}^{2}, \quad B_{n}(\cdot+\kappa)=B_{n}(\cdot) \quad \text { a.e. in } \mathbb{R}^{2},
$$

$B_{n}$ is equicoercive in $\mathbb{R}^{2}$, i.e.,

$$
\exists \alpha>0 \quad \text { such that } \quad \forall n \in \mathbb{N}, \forall \xi \in \mathbb{R}^{2}, \quad B_{n} \xi \cdot \xi \geq \alpha|\xi|^{2} \quad \text { a.e. in } \mathbb{R}^{2} .
$$


Let $\varepsilon_{n}$ be a sequence of positive numbers which tends to 0 . From the sequences $B_{n}$ and $\varepsilon_{n}$ we define the highly oscillating sequence of matrix-valued functions $A_{n}$ by

$$
A_{n}(x):=B_{n}\left(\frac{x}{\varepsilon_{n}}\right), \quad \text { a.e. } x \in \mathbb{R}^{2} .
$$

In virtue of (4.1) and (4.2) $A_{n}$ is an equicoercive sequence of $\varepsilon_{n}$-periodic matrix-valued functions in $L^{\infty}\left(\mathbb{R}^{2}\right)^{2 \times 2}$. Let $A_{n}^{*}$ be the constant matrix defined by

$$
A_{n}^{*} \lambda \cdot \lambda:=\min \left\{\int_{Y} B_{n}(y)(\lambda+\nabla \varphi(y)) \cdot(\lambda+\nabla \varphi(y)) d y: \varphi \in H_{\#}^{1}(Y)\right\}, \quad \lambda \in \mathbb{R}^{2},
$$

where $H_{\#}^{1}(Y)$ denotes the set of $Y$-periodic functions in $H_{\mathrm{loc}}^{1}\left(\mathbb{R}^{2}\right)$. The matrix $A_{n}^{*}$ is symmetric and positive definite with $A_{n}^{*} \geq \alpha I_{2}$. By the classical result of periodic homogenization (see e.g. [1]) $A_{n}^{*}$, for fixed $n$, is the homogenized matrix associated with the oscillating sequence $A_{n}\left(\frac{x}{\varepsilon}\right)$ as $\varepsilon$ tends to zero. Note that in the definition (4.3) of $A_{n}$ the oscillations period $\varepsilon_{n}$ depends on the sequence $n$.

In this periodic framework we are interested in the asymptotic behaviour of the diffusion energy $F_{n}$ defined by

$$
F_{n}(u):=\left\{\begin{array}{cl}
\int_{\Omega} A_{n} \nabla u \cdot \nabla u d x & \text { if } u \in H_{0}^{1}(\Omega) \\
+\infty & \text { if } u \in L^{2}(\Omega) \backslash H_{0}^{1}(\Omega),
\end{array}\right.
$$

as well as the conduction problem

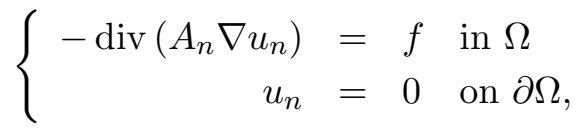

for a given $f$ in $H^{-1}(\Omega)$.

The following result shows that the asymptotic behaviour of the diffusion energy $F_{n}(4.5)$ only depends on the limit of the spectral radius $\rho\left(A_{n}^{*}\right)$ of the matrix $A_{n}^{*}(4.4)$.

Theorem 4.1. Let $\Omega$ be a bounded open set of $\mathbb{R}^{2}$. Consider a highly oscillating sequence of matrix-valued functions $A_{n}$ satisfying (4.1), (4.2) and (4.3). Then, we have the following alternative:

If $\rho\left(A_{n}^{*}\right)$ is bounded, there exists a subsequence, still denoted by $n$, and a positive definite matrix $A^{*}$ such that $A_{n}^{*}(4.4)$ converges to $A^{*}$ in $\mathbb{R}^{2 \times 2}$ and $F_{n}$ (4.5) $\Gamma$-converges for the strong topology of $L^{2}(\Omega)$ to the quadratic form $F$ associated with $A^{*}$ by

$$
F(u):=\left\{\begin{array}{cl}
\int_{\Omega} A^{*} \nabla u \cdot \nabla u d x & \text { if } u \in H_{0}^{1}(\Omega) \\
+\infty & \text { if } u \in L^{2}(\Omega) \backslash H_{0}^{1}(\Omega) .
\end{array}\right.
$$

If $\rho\left(A_{n}^{*}\right)$ tends to $+\infty$, the sequence $F_{n} \Gamma$-converges for the strong topology of $L^{2}(\Omega)$ to the quadratic form $F$ whose domain is

$$
D(F)=\{0\} .
$$

In term of the conduction problem (4.6) Theorem 4.1 implies the following result:

Corollary 4.2. Let $\Omega$ be a bounded open set of $\mathbb{R}^{2}$. Consider a highly oscillating sequence of matrix-valued functions $A_{n}$ satisfying (4.1), (4.2) and (4.3). Then, we have the following alternative: 
If $\rho\left(A_{n}^{*}\right)$ is bounded, there exists a subsequence, still denoted by $n$, and a positive definite matrix $A^{*}$ such that $A_{n}^{*}(4.4)$ converges to $A^{*}$ in $\mathbb{R}^{2 \times 2}$ and, for any $f$ in $H^{-1}(\Omega)$, the solution $u_{n}$ of (4.6) weakly converges in $H_{0}^{1}(\Omega)$ to the solution $u$ of the conduction problem

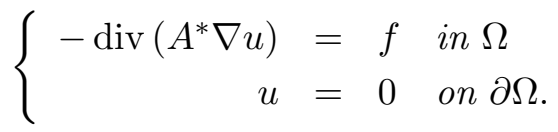

If $\rho\left(A_{n}^{*}\right)$ tends to $+\infty$, the sequence $u_{n}$ strongly converges to 0 in $H_{0}^{1}(\Omega)$.

Proof. Corollary 4.2 is a immediate consequence of Theorem 4.1 using the fact that the solution $u_{n}$ of (4.6) is the minimizer of the functional

$$
u \in L^{2}(\Omega) \longmapsto \frac{1}{2} F_{n}(u)-\int_{\Omega} f u d x,
$$

and the minimizers convergence property of the $\Gamma$-convergence (see e.g. Corollory 7.24 p. 84 of $[13])$.

Remark 4.3. Corollary 4.2 is an extension of the similar homogenization result obtained in [4] (by a complete different approach) under the assumption that the sequence of periodic matrix-valued $B_{n}(4.3)$ is bounded in $L^{1}(Y)^{2 \times 2}$. This condition is more restrictive since it is easy to check that the boundedness of $B_{n}$ in $L^{1}(Y)^{2 \times 2}$ implies the boundedness of $\rho\left(A_{n}^{*}\right)$. We can also build a periodic two-dimensional microstructure such that $\rho\left(A_{n}^{*}\right)$ is bounded while $\left\|B_{n}\right\|_{L^{1}(Y)^{2 \times 2}}$ is not. Therefore, Corollary 4.2 provides a complete answer to the periodic homogenization of the conduction problems with equicoercive sequences of symmetric conductivities.

\subsection{Proof of Theorem 4.1}

\section{The case where $\rho\left(A_{n}^{*}\right)$ is bounded}

Let $X_{n}^{i}, i=1,2$, be the unique function in $H_{\#}^{1}(Y)$, with zero $Y$-average value, solution of

$$
\forall \varphi \in H_{\#}^{1}(Y), \quad \int_{Y} B_{n} \nabla W_{n}^{i} \cdot \nabla \varphi d y=0, \quad \text { where } \quad W_{n}^{i}(y):=y_{i}+X_{n}^{i}(y),
$$

or equivalently,

$$
\operatorname{div}\left(B_{n}\left(e_{i}+\nabla X_{n}^{i}\right)\right)=0 \quad \text { in } \mathcal{D}^{\prime}\left(\mathbb{R}^{2}\right),
$$

where $\left(e_{1}, e_{2}\right)$ denotes the canonic basis of $\mathbb{R}^{2}$. Let $w_{n}^{i}$ be the highly oscillating sequence defined by

$$
w_{n}^{i}(x):=\varepsilon_{n} W_{n}^{i}\left(\frac{x}{\varepsilon_{n}}\right)=x_{i}+\varepsilon_{n} X_{n}^{i}\left(\frac{x}{\varepsilon_{n}}\right), \quad \text { for } x \in \Omega .
$$

By (4.11) and the definition (4.3) of $A_{n}$ the function $w_{n}^{i}$ is clearly $A_{n}$-harmonic. Moreover, by the $Y$-periodicity of $B_{n} \nabla W_{n}^{i} \cdot \nabla W_{n}^{i}$, by (4.10) and the definition (4.4) of $A_{n}^{*}$, we have for any bounded open subset $\omega$ of $\mathbb{R}^{2}$,

$$
\int_{\omega} A_{n} \nabla w_{n}^{i} \cdot \nabla w_{n}^{i} d x \leq c_{\omega} \int_{Y} B_{n} \nabla W_{n}^{i} \cdot \nabla W_{n}^{i} d y=c_{\omega} A_{n}^{*} e_{i} \cdot e_{i} \leq c<+\infty .
$$

This combined with the equicoerciveness of $A_{n}$ implies that the sequence $w_{n}^{i}$ is bounded in $H_{\text {loc }}^{1}\left(\mathbb{R}^{2}\right)$ and thus weakly converges to $x_{i}$ in $H_{\text {loc }}^{1}\left(\mathbb{R}^{2}\right)$. Then, thanks to Corollary 2.3 the sequence $w_{n}^{i}$ strongly converges to $x_{i}$ in $L^{\infty}(\Omega)$. On the other hand, by the boundedness assumption on $\rho\left(A_{n}^{*}\right)$ the sequence $A_{n}^{*}$ converges, up to a subsequence, to some constant 
matrix $A^{*}$ in the space $\mathbb{R}^{2 \times 2}$. Moreover, the $\varepsilon_{n}$-periodicity of $\nabla w_{n}^{i}$ implies that, for any $i, j=1,2$,

$$
A_{n} \nabla w_{n}^{i} \cdot \nabla w_{n}^{j}=\left(B_{n} \nabla W_{n}^{i} \cdot \nabla W_{n}^{j}\right)\left(\frac{x}{\varepsilon_{n}}\right) \rightarrow A^{*} e_{i} \cdot e_{j} \quad \text { weakly in } \mathcal{M}\left(\mathbb{R}^{2}\right) * .
$$

So, as the gradients of the functions $x_{i}, i \in\{1,2\}$, are independent at each point of $\Omega$, the sequences $w_{n}^{i}$ satisfy $(3.12)$ for any open disk contained in $\Omega$. Therefore, since $w_{n}^{i}$ are $A_{n}$-harmonic and converge uniformly in $\Omega$, the construction of Lemma 3.7 yields the measure $\mu$ and the matrix-valued $A$ by

$$
d \mu=\left(A^{*} e_{1} \cdot e_{1}+A^{*} e_{2} \cdot e_{2}\right) d x \quad \text { and } A e_{i} \cdot e_{j} d \mu=A^{*} e_{i} \cdot e_{j} d x
$$

hence $A^{*}=\left(A^{*} e_{1} \cdot e_{1}+A^{*} e_{2} \cdot e_{2}\right) A$. Then, in virtue of Theorem 3.4 the $\Gamma$-limit $F$ of the sequence $F_{n}(4.5)$ satisfies

$$
C_{c}^{1}(\Omega) \subset D(F) \quad \text { and } \quad F(u)=\int_{\Omega} A^{*} \nabla u \cdot \nabla u d x, \quad \forall u \in C_{c}^{1}(\Omega) .
$$

Let us conclude. On the one side, the equicoerciveness of $A_{n}$ and the lower semicontinuity of the $H_{0}^{1}(\Omega)$-norm give $D(F) \subset H_{0}^{1}(\Omega)$. On the other side, the density of $C_{c}^{1}(\Omega)$ in $H_{0}^{1}(\Omega)$, combined with the fact that $D(F)$ is a Hilbert space and that from (4.13) a sequence of $C_{c}^{1}(\Omega)$ which strongly converges in $H_{0}^{1}(\Omega)$ also strongly converges in $D(F)$, we get $D(F)=H_{0}^{1}(\Omega)$ and equality (4.13) holds true in $H_{0}^{1}(\Omega)$. Note that $F_{n} \Gamma$-converges to $F$ for the whole sequence such that $A_{n}^{*}$ converges to $A^{*}$ in $\mathbb{R}^{2 \times 2}$.

\section{The case where $\rho\left(A_{n}^{*}\right)$ tends to $+\infty$}

We proceed by contradiction. We assume that the domain $D(F)$ of the $\Gamma$-limit $F$ does not reduce to $\{0\}$. Then, we prove that $\rho\left(A_{n}^{*}\right)$ is necessarily bounded. To this end, we proceed in two steps. In the first step, we prove that there exists a continuous function in $D(F) \backslash\{0\}$. The second step is devoted to the proof of the boundedness of $\rho\left(A_{n}^{*}\right)$.

First step : $D(F) \cap C(\Omega) \neq\{0\}$.

Up to an extraction of a subsequence we can assume that the sequence $F_{n}$ defined by (4.5) $\Gamma$-converges to some quadratic functional $F: L^{2}(\Omega) \longrightarrow[0,+\infty]$. The starting assumption is that $D(F) \neq\{0\}$. Let $u \in D(F) \backslash\{0\}$. By the equicoerciveness of $A_{n}$ the function $u$ belongs to $H_{0}^{1}(\Omega)$. There exists a sequence $u_{n}$ in $H_{0}^{1}(\Omega)$ which strongly converges to $u$ in $L^{2}(\Omega)$ and such that $F_{n}\left(u_{n}\right)$ tends to $F(u)$. Up to enlarge the domain $\Omega$ and to extend the functions of $H_{0}^{1}(\Omega)$ by 0 outside $\Omega$, we may assume that the supports of $u, u_{n}$ are contained in a fixed compact $K$ of $\Omega$.

Firstly, let us prove that, for any $\tau \in \mathbb{R}^{2}$ of small enough norm, the translated function $u(\cdot+\tau)$ belongs to $D(F)$ and $F(u(\cdot+\tau))=F(u)$. We follow the procedure given in the proof of Theorem 24.1 of [13]. Let $\tau \in \mathbb{R}^{2}$ and let $\kappa_{n}$ be a sequence in $\mathbb{Z}^{2}$ such that $\tau_{n}:=\varepsilon_{n} \kappa_{n}$ tends to $\tau$. If $\tau$ has a small enough norm, then we have $K-\tau_{n} \subset \Omega$ for any $n \in \mathbb{N}$. Then, using successively the fact that $u_{n}\left(\cdot+\tau_{n}\right)$ is equal to 0 in $\Omega \backslash\left(K-\tau_{n}\right)$, the change of variable $y=x+\tau_{n}$ and the $\varepsilon_{n}$-periodicity of $A_{n}$, we obtain

$$
\begin{aligned}
F\left(u_{n}\left(\cdot+\tau_{n}\right)\right) & =\int_{K-\tau_{n}} A_{n}(x) \nabla u_{n}\left(x+\tau_{n}\right) \cdot \nabla u_{n}\left(x+\tau_{n}\right) d x \\
& =\int_{K} A_{n}(y) \nabla u_{n}(y) \cdot \nabla u_{n}(y) d y=F_{n}\left(u_{n}\right) .
\end{aligned}
$$

Moreover, the sequence $u_{n}\left(\cdot+\tau_{n}\right)$ strongly converges to $u(\cdot+\tau)$. Therefore, the $\Gamma$-liminf inequality implies that

$$
F(u(\cdot+\tau)) \leq \liminf _{n \rightarrow+\infty} F_{n}\left(u_{n}\left(\cdot+\tau_{n}\right)\right)=\liminf _{n \rightarrow+\infty} F_{n}\left(u_{n}\right)=F(u),
$$


which also yields $F(u) \leq F(u(\cdot+\tau-\tau)) \leq F(u(\cdot+\tau))$, and thus $F(u(\cdot+\tau))=F(u)$.

Secondly, let $\delta$ be a small enough positive number and let $v_{\delta}$ be the function defined on $\Omega$ by

$$
v_{\delta}(x):=\frac{1}{\delta^{2}} \int_{\delta Y} u(x+y) d y, \quad \text { for } x \in \Omega,
$$

which is continuous on $\Omega$. Let $\left(Q_{k}^{j}\right)_{1 \leq j \leq k}$, for $k \in \mathbb{N}^{*}$, be a covering of the set $\delta Y$ by $k$ squares of side $\frac{\delta}{\sqrt{k}}$ and let $y_{k}^{j}$ be the center of $Q_{k}^{j}$. Then, the sequence of convex combinations of translated of $u$ defined by

$$
v_{\delta}^{k}:=\frac{1}{\delta^{2}} \sum_{j=1}^{k}\left|Q_{k}^{j}\right| u\left(\cdot+y_{k}^{j}\right)
$$

strongly converges to $v_{\delta}$ in $L^{2}(\Omega)$ as $k \rightarrow+\infty$, for fixed $\delta$. Then, the lower semi-continuity and the convexity of $F$ yield

$$
F\left(v_{\delta}\right) \leq \liminf _{k \rightarrow+\infty} F\left(v_{\delta}^{k}\right) \leq \liminf _{k \rightarrow+\infty} \frac{1}{\delta^{2}} \sum_{j=1}^{k}\left|Q_{k}^{j}\right| F\left(u\left(\cdot+y_{k}^{j}\right)\right)=F(u)<+\infty .
$$

Therefore, $v_{\delta}$ belongs to $D(F) \cap C(\Omega)$. Since $v_{\delta}$ strongly converges to $u \neq 0$ in $L^{2}(\Omega), v_{\delta}$ is a non-zero function in $D(F) \cap C(\Omega)$ for $\delta$ small enough, which concludes the first step.

Second step : Boundedness of $\rho\left(A_{n}^{*}\right)$.

Let $v$ be a non-zero function in $D(F) \cap C(\Omega)$ and let $v_{n}$ be a sequence in $H_{0}^{1}(\Omega)$ which strongly converges to $v$ in $L^{2}(\Omega)$ and such that $F_{n}\left(v_{n}\right)$ tends to $F(v)$. By Theorem 2.1 the sequence $v_{n}$ uniformly converges to $v$ in $\Omega$. Since $v$ is a non-zero continuous function on $\Omega$, the uniform convergence of $v_{n}$ to $v$ implies that there exists a non-empty open subset $\omega_{0}$ of $\Omega$ and a constant $c_{0}>0$ such that

$$
\left|v_{n}(x)\right| \geq c_{0} \quad \text { a.e. } x \in \omega_{0} .
$$

Let $\lambda_{n}$ be a unit norm vector in $\mathbb{R}^{2}$ such that $A_{n}^{*} \lambda_{n} \cdot \lambda_{n}=\rho\left(A_{n}^{*}\right)$. Let $w_{n}$ be the highly oscillating sequence defined by

$$
w_{n}(x):=\lambda_{n} \cdot x+\varepsilon_{n} X_{n}\left(\frac{x}{\varepsilon_{n}}\right) \quad x \in \mathbb{R}^{2}, \quad \text { where } \quad X_{n}:=\left(\lambda_{n} \cdot e_{1}\right) X_{n}^{1}+\left(\lambda_{n} \cdot e_{2}\right) X_{n}^{2}
$$

and $X_{n}^{i}, i=1,2$, are the $Y$-periodic solutions of (4.11). Set $\tilde{Y}:=\left(-\frac{1}{2}, \frac{3}{2}\right)^{2}$. Since the function $W_{n}(y):=\lambda_{n} \cdot y+X_{n}(y)$ is $B_{n}$-harmonic in $\mathbb{R}^{2}$, by Corollary 2.5 there exists a constant $C>0$ such that for any $n \in \mathbb{N}$,

$$
\left\|W_{n}\right\|_{L^{\infty}(Y)} \leq C\left\|W_{n}\right\|_{H^{1}(\tilde{Y})} .
$$

Then, using the periodicity and the zero $Y$-average value of $X_{n}$, as well as the PoincaréWirtinger inequality yields

$$
\begin{aligned}
\left\|X_{n}\right\|_{L^{\infty}(Y)} & \leq 1+\left\|W_{n}\right\|_{L^{\infty}(Y)} \\
& \leq 1+\left\|\lambda_{n} \cdot y\right\|_{L^{\infty}(\tilde{Y})}+C\left\|X_{n}\right\|_{H^{1}(\tilde{Y})} \leq 1+\frac{3}{\sqrt{2}}+4 C\left\|X_{n}\right\|_{H^{1}(Y)} \\
& \leq C^{\prime}+C^{\prime}\left\|\nabla X_{n}\right\|_{L^{2}(Y)} \leq 2 C^{\prime}+C^{\prime}\left\|\nabla W_{n}\right\|_{L^{2}(Y)} .
\end{aligned}
$$

Moreover, the coerciveness of $B_{n}$ and the definition (4.4) of $A_{n}^{*}$ imply that

$$
\alpha\left\|\nabla W_{n}\right\|_{L^{2}(Y)^{2}}^{2} \leq \int_{Y} B_{n} \nabla W_{n} \cdot \nabla W_{n} d y=A_{n}^{*} \lambda_{n} \cdot \lambda_{n}=\rho\left(A_{n}^{*}\right),
$$


which combined with the previous estimates gives

$$
\left\|X_{n}\right\|_{L^{\infty}(Y)} \leq C^{\prime}+\frac{C^{\prime}}{\sqrt{\alpha}} \sqrt{\rho\left(A_{n}^{*}\right)} .
$$

Therefore, by the $Y$-periodicity of $X_{n}$ and the definition (4.15) of $w_{n}$ there exists a constant $c>0$ such that for any $n \in \mathbb{N}$,

$$
\left\|w_{n}\right\|_{L^{\infty}(\Omega)}^{2} \leq c+c \varepsilon_{n}^{2} \rho\left(A_{n}^{*}\right)
$$

On the other hand, using the $A_{n}$-harmonicity of $w_{n}(4.15)$ and the Cauchy-Schwarz inequality yields

$$
\begin{aligned}
\int_{\Omega} A_{n} \nabla w_{n} \cdot \nabla w_{n} v_{n}^{2} d x & =-2 \int_{\Omega} A_{n} \nabla w_{n} \cdot \nabla v_{n} v_{n} w_{n} d x \\
& \leq 2\left(\int_{\Omega} A_{n} \nabla w_{n} \cdot \nabla w_{n} v_{n}^{2} d x\right)^{\frac{1}{2}}\left(\int_{\Omega} A_{n} \nabla v_{n} \cdot \nabla v_{n} w_{n}^{2} d x\right)^{\frac{1}{2}}
\end{aligned}
$$

hence the inequality

$$
\int_{\Omega} A_{n} \nabla w_{n} \cdot \nabla w_{n} v_{n}^{2} d x \leq 4 \int_{\Omega} A_{n} \nabla v_{n} \cdot \nabla v_{n} w_{n}^{2} d x
$$

Let us conclude. On the one side, thanks to the uniform estimate (4.14) and the $\varepsilon_{n}$-periodicity of $A_{n} \nabla w_{n} \cdot \nabla w_{n}$ the left hand-side of (4.17) is bounded from below by a positive constant times

$$
\int_{\omega_{0}} A_{n} \nabla w_{n} \cdot \nabla w_{n} d x \geq c_{\omega_{0}} \int_{Y} B_{n} \nabla W_{n} \cdot \nabla W_{n} d y=c_{\omega_{0}} \rho\left(A_{n}^{*}\right)
$$

where $c_{\omega_{0}}$ is a positive constant only depending on $\omega_{0}$. On the other side, thanks to the uniform estimate (4.16) combined with the boundedness of $F_{n}\left(v_{n}\right)$ the right hand-side of (4.17) is bounded from above by

$$
c+c \varepsilon_{n}^{2} \rho\left(A_{n}^{*}\right)
$$

Therefore, there exists a constant $c>0$ such that for any $n \in \mathbb{N}$,

$$
\rho\left(A_{n}^{*}\right) \leq c+c \varepsilon_{n}^{2} \rho\left(A_{n}^{*}\right), \quad \text { with } \varepsilon_{n} \rightarrow 0,
$$

which implies that $\rho\left(A_{n}^{*}\right)$ is bounded. The proof of Theorem 4.1 is done.

Acknowledgment. The authors thank the referee for the improvement of Lemma 2.8.

\section{References}

[1] Bensoussan A., Lions J.L. \& Papanicolaou G., Asymptotic Analysis for Periodic Structures, North-Holland, 1978.

[2] M. Bellieud \& G. Bouchitté, "Homogenization of elliptic problems in a fiber reinforced structure. Nonlocal effects", Ann. Scuola Norm. Sup. Pisa Cl. Sci., 26 (4) (1998), 407-436.

[3] A. Beurling \& J. Deny, "Espaces de Dirichlet", Acta Matematica, 99 (1958), 203-224. 
[4] M. Briane, "Homogenization of high-conductivity periodic problems: Application to a general distribution of one-directional fibers", SIAM J. Math. Anal., 35 (1) (2003), 33-60.

[5] M. Briane, "Homogenization of non uniformly bounded operators: critical barrier for nonlocal effects", Arch. Rat. Mech. Anal., 164 (2002), 73-101.

[6] M. Briane, "Nonlocal effects in two-dimensional conductivity", Arch. Rat. Mech. Anal. 182 (2) (2006), 255-267.

[7] M. Briane \& J. Casado-Díaz, "Two-dimension div-curl results. Application to the lack of nonlocal effects in homogenization", to appear in Com. Part. Diff. Equa.

[8] M. Briane \& J. Casado-Díaz, "Uniform estimates for solutions of two-dimensional uniformly elliptic equations with unbounded coefficients", work in progress.

[9] M. Briane \& N. Tchou, "Fibered microstructures for some nonlocal Dirichlet forms", Ann. Scuola Norm. Sup. Pisa Cl. Sci., 30 (4) (2001), 681-711.

[10] G. Buttazzo \& G. Dal Maso, "T-limits of integral functionals", J. Analyse Math., 37 (1980), 145-185.

[11] M. Camar-Eddine \& P. Seppecher, "Closure of the set of diffusion functionals with respect to the Mosco-convergence", Math. Models Methods Appl. Sci., 12 (8) (2002), 1153-1176.

[12] L. Carbone \& C. Sbordone, "Some properties of $\Gamma$-limits of integral functionals", Ann. Mate. Pura Appl., 122 (1979), 1-60.

[13] G. Dal Maso, An introduction to Г-convergence, Birkhaüser, Boston, 1993.

[14] L.C. Evans, Weak convergence methods for nonlinear partial differential equations, AMS, Providence (1990).

[15] V.N. Fenchenko \& E.YA. Khruslov, "Asymptotic of solution of differential equations with strongly oscillating matrix of coefficients which does not satisfy the condition of uniform boundedness", Dokl. AN Ukr.SSR, 4 (1981).

[16] D. Gilbarg \& N. Trudinger, Elliptic Partial Differential Equations of Second Order, Springer, 1977.

[17] E.YA. Khruslov, "Homogenized models of composite media", Composite Media and Homogenization Theory, ed. by G. Dal Maso and G.F. Dell'Antonio, in Progress in Nonlinear Differential Equations and Their Applications, Birkhaüser (1991), 159182.

[18] U. Mosco, "Composite media and asymptotic Dirichlet forms", J. of Functional Analysis, 123 (2) (1994), 368-421.

[19] J. Mossino, Inégalités Isopérimétriques et Applications en Physique, Hermann, Paris, 1984.

[20] F. Murat, "H-convergence", Séminaire d'Analyse Fonctionnelle et Numérique, 1977-78, Université d'Alger, multicopied, 34 pp. English translation : F. MURAT \& L. TARTAR, "H-convergence", Topics in the Mathematical Modelling of Composite Materials, ed. by L. Cherkaev \& R.V. Kohn, Progress in Nonlinear Differential Equations and their Applications, 31, Birkaüser, Boston, 1998, 21-43.

[21] F. Murat, "Compacité par compensation", Ann. Scuola Norm. Sup. Pisa Cl. Sci., 5 (4) (1978), 489-507.

[22] F. Pólya \& G. Szegö, Isoperimetric Inequalities, Princeton University Press, Princeton, 1951. 
[23] S. Spagnolo, "Sulla convergenza di soluzioni di equazioni paraboliche ed ellittiche", Ann. Scuola Norm. Sup. Pisa Cl. Sci., 22 (3) (1968), 571-597.

[24] G. Talenti, "Best constant in Sobolev inequality", Ann. Mat. Pura. Appl., 110 (4) (1976), 353-372.

[25] L. Tartar, Cours Peccot, Collège de France (1977), partly written in [20].

[26] L. TARTAR, "Compensated compactness and applications to partial differential equations, nonlinear analysis and mechanics", Heriot-Watt symposium IxV, Pitman, San Francisco (1979), 136-212. 